\title{
Classification of finite-multiplicity symmetric pairs
}

\author{
In honour of Professor Dynkin for his 90th birthday
}

Toshiyuki KOBAYASHI * and Toshihiko MATSUKI ${ }^{\dagger}$

\begin{abstract}
We give a complete classification of the reductive symmetric pairs $(G, H)$ for which the homogeneous space $(G \times H) / \operatorname{diag} H$ is real spherical in the sense that a minimal parabolic subgroup has an open orbit. Combining with a criterion established in [T. Kobayashi-T. Oshima, Adv. Math. 2013], we give a necessary and sufficient condition for a reductive symmetric pair $(G, H)$ such that the multiplicities for the branching law of the restriction of any admissible smooth representation of $G$ to $H$ have finiteness/boundedness property.
\end{abstract}

Keywords: branching law, restriction of representation, reductive group, real spherical variety, symmetric pair, admissible representations.

MSC 2010; primary 22E46; secondary 14M15, 53C35.

\section{Contents}

1 Introduction and statement of main results 2

2 Strategy of the proof 6

3 Linearization of the open-orbit conditions (PP) and (QP) 9

3.1 Parabolic subgroup $Q$ associated to $(G, H) \ldots \ldots$

3.2 Criterion for $(\mathrm{PP})$ and $(\mathrm{QP}) \ldots \ldots \ldots \ldots$. . . . . . . . . . . . . . . .

$3.3 c$-dual of symmetric pairs and $(\mathrm{QP}) \ldots \ldots \ldots$. . . . . . . . . . 14

3.4 Further properties for $(\mathrm{QP}) \ldots \ldots \ldots \ldots$

\footnotetext{
${ }^{*}$ Kavli IPMU (WPI) and Graduate School of Mathematical Sciences, the University of Tokyo, Meguro-ku, Tokyo, 153-8914, Japan, E-mail address: toshi@ms.u-tokyo.ac.jp

${ }^{\dagger}$ Faculty of Letters, Ryukoku University, Kyoto, 612-8577, Japan, E-mail address: matsuki@let.ryukoku.ac.jp
} 
5 Some classical and exceptional cases $\quad 20$

$5.1(G, H)=(U(i+j, k+l ; \mathbb{F}), U(i, k ; \mathbb{F}) \times U(j, l ; \mathbb{F})) \ldots \ldots \ldots$

$5.2 \quad(G, H)=(G L(p+q, \mathbb{F}), G L(p, \mathbb{F}) \times G L(q, \mathbb{F})) \ldots \ldots \ldots$

$5.3(G, H)=(O(m+n, \mathbb{C}), O(m, \mathbb{C}) \times O(n, \mathbb{C})) \ldots \ldots \ldots$

$5.4(G, H)=\left(O^{*}(2 p+2 q), O^{*}(2 p) \times O^{*}(2 q)\right) \ldots \ldots \ldots \ldots \ldots$

$5.5(G, H)$ is of type $\left(C_{n}, A_{n}\right) \ldots \ldots \ldots \ldots \ldots \ldots$

$5.6(G, H)=(S p(p+q, \mathbb{F}), S p(p, \mathbb{F}) \times S p(q, \mathbb{F})), \mathbb{F}=\mathbb{R}$ or $\mathbb{C} \ldots \ldots$

$5.7 \quad(G, H)=\left(O^{*}(2 p+2 q), U(p, q)\right) \ldots \ldots \ldots \ldots \ldots$

$5.8(\mathfrak{g}, \mathfrak{h})=\left(\mathfrak{e}_{6(-26)}, \mathfrak{s o}(9,1)+\mathbb{R}\right) \ldots \ldots \ldots \ldots \ldots$

6 Symmetric pair $(G, H)$ with $\operatorname{rank}_{\mathbb{R}} H=1$

7 Associated symmetric pairs of non- $K_{\varepsilon}$-family 35

8 Associated symmetric pairs of $K_{\varepsilon}$-family 37

9 Applications to branching problems 40

9.1 Admissible smooth representations . . . . . . . . . . . . . 41

9.2 Finite multiplicity property in branching laws $\ldots \ldots \ldots \ldots$

9.3 Uniformly bounded multiplicities . . . . . . . . . . . . . 43

9.4 Invariant trilinear forms $\ldots \ldots \ldots \ldots \ldots$

\section{Introduction and statement of main results}

A complex manifold $X_{\mathbb{C}}$ with action of a complex reductive group $G_{\mathbb{C}}$ is called spherical if a Borel subgroup of $G_{\mathbb{C}}$ has an open orbit in $X_{\mathbb{C}}$. In the real setting, in search of a good framework for global analysis on homogeneous spaces which are broader than the usual (e.g. symmetric spaces), we advocated in [10] the importance of an analogous notion for real reductive groups $G$ and proposed to call:

Definition 1.1. We say a smooth manifold $X$ with $G$-action is real spherical if a minimal parabolic subgroup $P_{G}$ of $G$ has an open orbit in $X$.

In the case where $G$ acts transitively on $X, P_{G}$ has finitely many orbits in $X$ if $X$ is real spherical (see [13, Remark 2.5 (4)] and references therein).

Suppose that $H$ is a closed subgroup which is reductive in $G$. Let $P_{H}$ be a minimal parabolic subgroup of $H$. 
Definition $1.2(13)$. We say the pair $(G, H)$ satisfies $(\mathrm{PP})$ if one of the following four equivalent conditions is satisfied.

(PP1) $(G \times H) / \operatorname{diag} H$ is real spherical as a $(G \times H)$-space.

(PP2) $G / P_{H}$ is real spherical as a $G$-space.

(PP3) $G$ has an open orbit in $G / P_{G} \times G / P_{H}$ via the diagonal action.

(PP4) There are finitely many $G$-orbits in $G / P_{G} \times G / P_{H}$ via the diagonal action.

The above four equivalent conditions are determined only by the Lie algebras $\mathfrak{g}$ and $\mathfrak{h}$ of the Lie groups $G$ and $H$, respectively. Therefore we also say that the pair $(\mathfrak{g}, \mathfrak{h})$ of Lie algebras satisfies (PP).

A natural question is to find all the pairs $(\mathfrak{g}, \mathfrak{h})$ of real reductive Lie algebras satisfying (PP) when $\mathfrak{h}$ is maximal reductive in $\mathfrak{g}$.

We say $(G, H)$ is a reductive symmetric pair if $H$ is an open subgroup of the fixed point subgroup $G^{\sigma}$ of some involutive automorphism $\sigma$ of $G$. Reductive symmetric pairs $(G, H)$ give typical examples of maximal reductive subalgebras $\mathfrak{h}$ in $\mathfrak{g}$, and provide important setups in branching laws of the restriction $G \downarrow H$.

The main goal of this paper is to establish a complete classification of reductive symmetric pairs $(G, H)$ having the geometric condition $(\mathrm{PP})$.

Theorem 1.3. Suppose $(G, H)$ is a reductive symmetric pair. Then the following two conditions are equivalent:

(i) $(G, H)$ satisfies $(\mathrm{PP})$, namely, $(G \times H) / \operatorname{diag} H$ is real spherical.

(ii) The pair $(\mathfrak{g}, \mathfrak{h})$ of the Lie algebras is isomorphic (up to outer automorphisms) to the direct sum of the following pairs:

A) Trivial case: $\mathfrak{g}=\mathfrak{h}$.

B) Abelian case: $\mathfrak{g}=\mathbb{R}, \mathfrak{h}=\{0\}$.

C) Compact case: $\mathfrak{g}$ is the Lie algebra of a compact simple Lie group.

D) Riemannian symmetric pair: $\mathfrak{h}$ is the Lie algebra of a maximal compact subgroup $K$ of a non-compact simple Lie group $G$.

E) Split rank one case $\left(\operatorname{rank}_{\mathbb{R}} G=1\right)$ :
E1) $(\mathfrak{o}(p+q, 1), \mathfrak{o}(p)+\mathfrak{o}(q, 1))$
$(p+q \geq 2)$.
E2) $(\mathfrak{s u}(p+q, 1), \mathfrak{s}(\mathfrak{u}(p)+\mathfrak{u}(q, 1)))$
$(p+q \geq 1)$. 
E3) $(\mathfrak{s p}(p+q, 1), \mathfrak{s p}(p)+\mathfrak{s p}(q, 1)) \quad(p+q \geq 1)$.

E4) $\left(\mathfrak{f}_{4(-20)}, \mathfrak{o}(8,1)\right)$.

F) Strong Gelfand pairs and their real forms:

F1) $(\mathfrak{s l}(n+1, \mathbb{C}), \mathfrak{g l}(n, \mathbb{C})) \quad(n \geq 2)$.

F2) $(\mathfrak{o}(n+1, \mathbb{C}), \mathfrak{o}(n, \mathbb{C})) \quad(n \geq 2)$.

F3) $(\mathfrak{s l}(n+1, \mathbb{R}), \mathfrak{g l}(n, \mathbb{R})) \quad(n \geq 1)$.

F4) $(\mathfrak{s u}(p+1, q), \mathfrak{u}(p, q)) \quad(p+q \geq 1)$.

F5) $(\mathfrak{o}(p+1, q), \mathfrak{o}(p, q)) \quad(p+q \geq 2)$.

G) $(\mathfrak{g}, \mathfrak{h})=\left(\mathfrak{g}^{\prime}+\mathfrak{g}^{\prime}, \operatorname{diag} \mathfrak{g}^{\prime}\right)$ Group case:

G1) $\mathfrak{g}^{\prime}$ is the Lie algebra of a compact simple Lie group.

G2) $(\mathfrak{o}(n, 1)+\mathfrak{o}(n, 1), \operatorname{diag} \mathfrak{o}(n, 1)) \quad(n \geq 2)$.

H) Other cases:
H1) $(\mathfrak{o}(2 n, 2), \mathfrak{u}(n, 1))$
$(n \geq 1)$.
H2) $\left(\mathfrak{s u}^{*}(2 n+2), \mathfrak{s u}(2)+\mathfrak{s u}^{*}(2 n)+\mathbb{R}\right) \quad(n \geq 1)$.
H3) $\left(\mathfrak{o}^{*}(2 n+2), \mathfrak{o}(2)+\mathfrak{o}^{*}(2 n)\right)$
$(n \geq 1)$.
H4) $(\mathfrak{s p}(p+1, q), \mathfrak{s p}(p, q)+\mathfrak{s p}(1))$.
H5) $\left(\mathfrak{e}_{6(-26)}, \mathfrak{s o}(9,1)+\mathbb{R}\right)$.

In the above description of the classification, we do not intend to write irreducible symmetric pairs in an exclusive way. Indeed some of the above pairs are isomorphic to each other when $\mathfrak{g}$ is of small dimension. For instance, $(\mathrm{E} 1)$ with $(p, q)=(4,1)$ is isomorphic to (H2) with $n=1$, namely,

$$
(\mathfrak{o}(5,1), \mathfrak{o}(4)+\mathfrak{o}(1,1)) \simeq\left(\mathfrak{s u} \mathfrak{u}^{*}(4), \mathfrak{s u}(2)+\mathfrak{s u}^{*}(2)+\mathbb{R}\right)
$$

Remark 1.4. It would be interesting to give a complete list of the pairs $(\mathfrak{g}, \mathfrak{h})$ of reductive Lie algebras having the property (PP) by dropping the assumption that $(\mathfrak{g}, \mathfrak{h})$ is a symmetric pair. (Cf. Dynkin [5] for the description of maximal reductive Lie algebras in simple Lie algebras over $\mathbb{C}$.) In view of the classification in Theorem 1.3 it is plausible that there are not many non-symmetric pairs $(\mathfrak{g}, \mathfrak{h})$ satisfying $(\mathrm{PP})$ if $H$ is noncompact.

Next we also consider another property, to be denoted by (BB), which is stronger than (PP). For this, suppose further that $G$ is an algebraic reductive group and $H$ is a reductive subgroup defined algebraically over $\mathbb{R}$. Let $G_{\mathbb{C}}$ be a complex Lie group 
with Lie algebra $\mathfrak{g}_{\mathbb{C}}=\mathfrak{g} \otimes_{\mathbb{R}} \mathbb{C}$, and $H_{\mathbb{C}}$ a subgroup of $G_{\mathbb{C}}$ with complexified Lie algebra $\mathfrak{h}_{\mathbb{C}}=\mathfrak{h} \otimes_{\mathbb{R}} \mathbb{C}$. Let $B_{G}$ and $B_{H}$ be Borel subgroups of $G_{\mathbb{C}}$ and $H_{\mathbb{C}}$, respectively.

Definition 1.5. We say the pair $(G, H)$ (or the pair $(\mathfrak{g}, \mathfrak{h})$ ) satisfies (BB) if one of the following equivalent conditions is satisfied:

(BB1) $\left(G_{\mathbb{C}} \times H_{\mathbb{C}}\right) / \operatorname{diag} H_{\mathbb{C}}$ is spherical as a $\left(G_{\mathbb{C}} \times H_{\mathbb{C}}\right)$-space.

(BB2) $G_{\mathbb{C}} / B_{H}$ is spherical as a $G_{\mathbb{C}^{-} \text {-space. }}$

(BB3) $G_{\mathbb{C}}$ has an open orbit in $G_{\mathbb{C}} / B_{G} \times G_{\mathbb{C}} / B_{H}$ via the diagonal action.

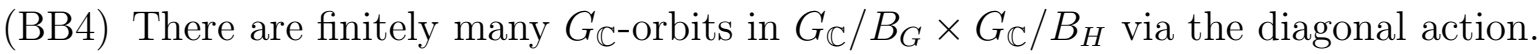

It follows from [13, Lemmas 4.2 and 5.3] that we have the implication

$$
(\mathrm{BB}) \Rightarrow(\mathrm{PP})
$$

Among the pairs $(\mathfrak{g}, \mathfrak{h})$ in Theorem 1.3 satisfying $(\mathrm{PP})$, we list the pairs $(\mathfrak{g}, \mathfrak{h})$ satisfying $(\mathrm{BB})$ as follows:

Proposition 1.6. Suppose $(\mathfrak{g}, \mathfrak{h})$ is a reductive symmetric pair. Then the following conditions are equivalent:

(i) $(\mathfrak{g}, \mathfrak{h})$ satisfies $(\mathrm{BB})$.

(ii) The pair of the Lie algebras $(\mathfrak{g}, \mathfrak{h})$ is isomorphic (up to outer automorphisms) to the direct sum of pairs $(\mathrm{A}),(\mathrm{B})$ and $(\mathrm{F} 1)-(\mathrm{F} 5)$.

Remark 1.7. The classification in Theorem 1.3 (or in the "irreducible case", see Theorem 2.1) was known earlier in the following special cases:

1) $(\mathfrak{g}, \mathfrak{h})$ complex pairs: $\quad(\mathrm{BB}) \Leftrightarrow(\mathrm{F} 1)$ or $(\mathrm{F} 2) \quad(\mathrm{M}$. Krämer [17]).

2) $\operatorname{rank}_{\mathbb{R}} G=1:(\mathrm{PP}) \Leftrightarrow(\mathrm{E} 1)-(\mathrm{E} 4) \quad(\mathrm{B}$. Kimelfeld $[8])$.

2) $(\mathfrak{g}, \mathfrak{h})=\left(\mathfrak{g}^{\prime}+\mathfrak{g}^{\prime}, \operatorname{diag} \mathfrak{g}^{\prime}\right)($ "group case"): $\quad(\mathrm{PP}) \Leftrightarrow(\mathrm{G} 1)$ or $(\mathrm{G} 2) \quad$ (匹10 $)$.

Concerning Remark 1.7 (2), neither the concept (PP) nor a minimal parabolic subgroup of $H$ appeared in [8], but one might read (E1)-(E4) from his work. The case (1) ("strong Gelfand pairs") was studied in connection with finite-dimensional representations of compact Lie groups, and the "group case" (3) with the tensor product of two (infinite-dimensional) representations, see Corollary 9.6 for more details.

The significance of these geometric conditions $(\mathrm{PP})$ and $(\mathrm{BB})$ is their applications to branching problems of infinite-dimensional representations of real reductive groups $G$ to subgroups $H$ : 
Fact 1.8 ([13, Theorems $\mathrm{C}$ and $\mathrm{D}])$. Suppose $G$ is a real reductive Lie group, and $H$ a reductive subgroup defined algebraically over $\mathbb{R}$.

1) (Finite-multiplicity for branching) The pair $(G, H)$ satisfies $(\mathrm{PP})$ if and only if

$$
\operatorname{dim} \operatorname{Hom}_{H}\left(\left.\pi\right|_{H}, \tau\right)<\infty
$$

for any admissible smooth representation $\pi$ of $G$ and for any admissible smooth representation $\tau$ of $H$.

2) (Bounded-multiplicity for branching) The pair $(G, H)$ satisfies (BB) if and only if there exists a constant $C<\infty$ such that

$$
\operatorname{dim} \operatorname{Hom}_{H}\left(\left.\pi\right|_{H}, \tau\right) \leq C
$$

for any irreducible smooth representation $\pi$ of $G$ and for any irreducible smooth representation $\tau$ of $H$.

In Section 9 we review briefly some basic notion on admissible smooth representations of real reductive groups and discuss applications of our classification results to branching problems in details.

Organization of the paper. We give an outline of the proof of Theorem $1.3 \mathrm{in}$ Section 2 by dividing it into five steps. Sections 3 to 8 are devoted to the proof of Theorem 1.3 of the paper.

In Section 9 we explain our initial motivation for studying the real spherical property (PP) from the viewpoint of the (infinite-dimensional) representation theory of real reductive groups, and give an application of our geometric results to branching problems of smooth admissible representations.

Notation: $\mathbb{R}_{+}:=\{t \in \mathbb{R}: t>0\}$, and $\mathbb{R}_{\geq 0}:=\{t \in \mathbb{R}: t \geq 0\}$

Acknowledgement: The first author was partially supported by Grant-in-Aid for Scientific Research (A)(25247006) JSPS.

\section{Strategy of the proof}

We give an outline of the proof of Theorem 1.3 by dividing it into five steps.

Step 1. Reduction to irreducible symmetric pairs.

A reductive symmetric pair $(\mathfrak{g}, \mathfrak{h})$ is said to be irreducible if $\mathfrak{g} \neq ⿻ \mathbb{R}, \mathfrak{h}$ and if $(\mathfrak{g}, \mathfrak{h})$ is not isomorphic to the direct sum of two reductive symmetric pairs $\left(\mathfrak{g}_{1}, \mathfrak{h}_{1}\right)$ and $\left(\mathfrak{g}_{2}, \mathfrak{h}_{2}\right)$. 
The proof of Theorem 1.3 reduces to the case where $(\mathfrak{g}, \mathfrak{h})$ is an irreducible symmetric pair. This consists of two families up to outer automorphisms:

1) (group case) $\left(\mathfrak{g}^{\prime}+\mathfrak{g}^{\prime}, \operatorname{diag} \mathfrak{g}^{\prime}\right)$ with $\mathfrak{g}^{\prime}$ simple.

2) $(\mathfrak{g}, \mathfrak{h})$ with $\mathfrak{g}$ simple.

Therefore the task of this article is to carry out the following classification:

Theorem 2.1. For an irreducible symmetric pair $(\mathfrak{g}, \mathfrak{h})$, the following two conditions are equivalent:

(i) $(G \times H) / \operatorname{diag} H$ is real spherical.

(ii) $(\mathfrak{g}, \mathfrak{h})$ is isomorphic to one of $(\mathrm{C})-(\mathrm{H})$ up to outer automorphisms.

The main case is when $\mathfrak{g}$ is simple. The "group case" $(\mathrm{G})$ is relatively easy and the classification of those satisfying (PP) was already given in [10], but we supply a proof here for the sake of completeness.

Step 2. Condition (QP).

Suppose $\sigma$ is an involutive automorphism of $G$. In general there is no $\sigma$-stable minimal parabolic subgroup of $G$. We introduce a condition (QP) which is slightly weaker than (PP) by using a $\sigma$-stable parabolic subgroup (Subsection 3.1). The difference between (QP) and (PP) is described as in Theorem 2.2 below.

Step 3. Linearization of (PP) and (QP).

We find a necessary and sufficient condition for a pair $(G, H)$ to satisfy the conditions (PP) (and also (QP)), by means of the open-orbit property of a certain linear action (Theorems 3.3 and 3.4). The case (QP) is easier because the parabolic subgroup $Q$ is $\sigma$-stable, whereas the criterion of $(\mathrm{PP})$ is more involved since the parabolic subgroup $P_{G}$ (or its conjugate) is not necessarily $\sigma$-stable.

Step 4. The proof for (ii) $\Rightarrow$ (i) in Theorem 1.3.

The proof is carried out in Sections 4,5 and 6. We shall verify the existence of an open orbit of the adjoint action of $\left(M_{H} \cap M_{G}\right) A_{H}$ in $\mathfrak{n}^{-\sigma}$, by using the criterion of (PP) in Step 3.

Section 5 deals with specific symmetric pairs in a case-by-case fashion, for instance, $(\mathfrak{g}, \mathfrak{h})=(\mathfrak{o}(i+j, k+l), \mathfrak{o}(i, k)+\mathfrak{o}(j, l))$. We use the invariant theory of quivers (Subsection 5.1). In the section we classify not only the irreducible symmetric pairs $(\mathfrak{g}, \mathfrak{h})$ satisfying (PP) but also those satisfying (QP). See Theorem 2.2 below. Here is the 
precise place where the proof for (ii) $\Rightarrow$ (i) in Theorem 1.3 is given. We give two proofs for some families of symmetric pairs $(G, H)$.

$\begin{array}{ll}\text { Proposition 4.3 } & (\mathrm{F} 1)(\mathrm{F} 2)(\mathrm{F} 3)(\mathrm{F} 4)(\mathrm{F} 5) \\ \text { Proposition 5.1 } & (\mathrm{E} 1)(\mathrm{E} 2)(\mathrm{E} 3)(\mathrm{F} 4)(\mathrm{F} 5)(\mathrm{H} 4) \\ \text { Proposition 5.4 } & (\mathrm{F} 1)(\mathrm{F} 3)(\mathrm{H} 2) \\ \text { Proposition 5.6 } & (\mathrm{F} 2) \\ \text { Proposition 5.7 } & (\mathrm{H} 3) \\ \text { Proposition 5.14 } & (\mathrm{H} 5) \\ \text { Proposition 6.2 } & (\mathrm{E} 1)(\mathrm{E} 2)(\mathrm{E} 3)(\mathrm{E} 4)\end{array}$

Step 5. The proof for (i) $\Rightarrow$ (ii) in Theorem 1.3 .

The proof is carried out together with the classification of a larger set of the irreducible symmetric pairs satisfying (QP). We divide irreducible symmetric pairs $(\mathfrak{g}, \mathfrak{h})$ into the following three cases. Some of the results for concrete examples in Section 5 are used in Sections 7 and 8 .

Case 5a. (Section 6) $\operatorname{rank}_{\mathbb{R}} H=1$.

Case 5b. (Section 7) $\quad \operatorname{rank}_{\mathbb{R}} H \geq 2, \quad\left(\mathfrak{g}, \mathfrak{g}^{\sigma \theta}\right)$ does not belong to $K_{\varepsilon}$-family.

Case 5c. (Section 8) $\quad \operatorname{rank}_{\mathbb{R}} H \geq 2, \quad\left(\mathfrak{g}, \mathfrak{g}^{\sigma \theta}\right)$ belongs to $K_{\varepsilon}$-family.

As a byproduct of the proof of Theorem 1.3, we obtain a complete list of the irreducible symmetric pairs $(\mathfrak{g}, \mathfrak{h})$ satisfying $(\mathrm{QP})$ :

Theorem 2.2. Irreducible symmetric pairs satisfying (QP) but not satisfying $(\mathrm{PP})$ are listed as follows:

$$
\begin{aligned}
\mathrm{I}_{\mathbb{R}}:(\mathfrak{o}(p+1, q), \mathfrak{o}(p)+\mathfrak{o}(1, q)) & (p, q \geq 2), \\
\mathrm{I}_{\mathbb{C}}:(\mathfrak{s u}(p+1, q), \mathfrak{s}(\mathfrak{u}(p)+\mathfrak{u}(1, q))) & (p, q \geq 2), \\
\mathrm{I}_{\mathbb{H}}:(\mathfrak{s p}(p+1, q), \mathfrak{s p}(p)+\mathfrak{s p}(1, q)) & (p, q \geq 2) \\
\text { II }:(\mathfrak{o}(n+1, \mathbb{C}), \mathfrak{o}(n, 1)) & (n \geq 4) \\
\text { III }:\left(\mathfrak{o}^{*}(2 n+2), \mathfrak{u}(n, 1)\right) & (n \geq 4) .
\end{aligned}
$$

Outline of proof. We shall see in Proposition 6.2 that $(\mathfrak{g}, \mathfrak{h})$ satisfies (QP) if $(\mathfrak{g}, \mathfrak{h})$ is one of $I_{\mathbb{R}}, I_{\mathbb{C}}, I_{\mathbb{H}}$, II or III. Parts of this assertion also follow from Proposition 5.1 when $(\mathfrak{g}, \mathfrak{h})$ is $\mathrm{I}_{\mathbb{R}}, \mathrm{I}_{\mathbb{C}}$, or $\mathrm{I}_{\mathbb{H}}$, and from Proposition 5.13 when $(\mathfrak{g}, \mathfrak{h})$ is III. The exhaustion of this list is a crucial part of Step 5 . 
By the classification in Theorem 2.2, we obtain

Corollary 2.3. For irreducible symmetric pairs $(\mathfrak{g}, \mathfrak{h})$ with $\operatorname{rank}_{\mathbb{R}} \mathfrak{h} \geq 2,(\mathrm{PP}) \Leftrightarrow(\mathrm{QP})$.

\section{Linearization of the open-orbit conditions (PP) and $(\mathrm{QP})$}

The goal of this section is to give a criterion for (PP) by linearization. The main result is Theorem 3.3. The proof for the implication (ii) $\Rightarrow$ (i) in Theorem 2.1 is carried out by this criterion in later sections. In order to optimise the proof for the exhaustion of the list $(\mathrm{C})-(\mathrm{H})$, we introduce another geometric condition (QP), which is slightly weaker than (PP). Then the condition (QP) becomes a stepping-stone in the proof of the implication (i) $\Rightarrow$ (ii) by removing most of the symmetric pairs $(\mathfrak{g}, \mathfrak{h})$ that do not satisfy (PP). The condition (QP) is also linearized in Theorem 3.4. We shall further analyze the condition (QP) in Propositions 3.9 and 3.11 .

\subsection{Parabolic subgroup $Q$ associated to $(G, H)$}

Let $G$ be a real reductive linear Lie group. Suppose that $\sigma$ is an involutive automorphism of $G$. The set $G^{\sigma}:=\{g \in G: \sigma g=g\}$ of fixed points by $\sigma$ is a closed subgroup of $G$. We say $(G, H)$ is a reductive symmetric pair if $H$ is an open subgroup of $G^{\sigma}$ for some $\sigma$.

We take a Cartan involution $\theta$ of $G$ commuting with $\sigma$, and set $K:=G^{\theta}$, a maximal compact subgroup of $G$. The Lie algebras will be denoted by lower German letters such as $\mathfrak{g}, \mathfrak{h}, \mathfrak{k}, \cdots$, and we shall use the same letters $\sigma$ and $\theta$ for the induced automorphisms of the Lie algebra $\mathfrak{g}$. If $\tau$ is an involutive endomorphism of a real vector space $V$, then $\tau$ is diagonalizable with eigenvalues \pm 1 . We write the eigenspace decomposition as

$$
V=V^{\tau}+V^{-\tau}
$$

where $V^{ \pm \tau}:=\{X \in V: \tau X= \pm X\}$. With the above notation, $\mathfrak{h}=\mathfrak{g}^{\sigma}, \mathfrak{k}=\mathfrak{g}^{\theta}$, and $\mathfrak{g}=\mathfrak{g}^{\theta}+\mathfrak{g}^{-\theta}$ is a Cartan decomposition of the Lie algebra $\mathfrak{g}$.

We fix a maximal abelian subspace $\mathfrak{a}_{H}$ in $\mathfrak{h}^{-\theta}$, and extend it to a maximal abelian subspace $\mathfrak{a}_{G}$ in $\mathfrak{g}^{-\theta}$. The split rank of $H$ will be denoted by

$$
\operatorname{rank}_{\mathbb{R}} H:=\operatorname{dim}_{\mathbb{R}} \mathfrak{a}_{H}
$$

For $\alpha \in \mathfrak{a}_{G}^{*}$, we write

$$
\mathfrak{g}\left(\mathfrak{a}_{G} ; \alpha\right):=\left\{X \in \mathfrak{g}:[H, X]=\alpha(H) X \quad \text { for } \quad H \in \mathfrak{a}_{G}\right\},
$$


and denote by $\Sigma\left(\mathfrak{g}, \mathfrak{a}_{G}\right)$ the set of nonzero $\alpha$ such that $\mathfrak{g}\left(\mathfrak{a}_{G} ; \alpha\right) \neq\{0\}$. Similar notation is applied to $\mathfrak{a}_{H}$. Then the set of nonzero weights $\Sigma\left(\mathfrak{g}, \mathfrak{a}_{H}\right)$ satisfies the axiom of root systems ([19, Theorem 2.1]) as well as $\Sigma\left(\mathfrak{g}, \mathfrak{a}_{G}\right)$. We choose compatible positive systems $\Sigma^{+}\left(\mathfrak{g}, \mathfrak{a}_{G}\right)$ and $\Sigma^{+}\left(\mathfrak{g}, \mathfrak{a}_{H}\right)$ in the sense that

$$
\left.\alpha\right|_{\mathfrak{a}_{H}} \in \Sigma^{+}\left(\mathfrak{g}, \mathfrak{a}_{H}\right) \cup\{0\} \text { for any } \alpha \in \Sigma^{+}\left(\mathfrak{g}, \mathfrak{a}_{G}\right)
$$

We write $\mathfrak{g}\left(\mathfrak{a}_{G} ; \alpha\right)$ and $\mathfrak{g}\left(\mathfrak{a}_{H} ; \lambda\right)$ for the root space of $\alpha \in \Sigma\left(\mathfrak{g}, \mathfrak{a}_{G}\right)$ and $\lambda \in \Sigma\left(\mathfrak{g}, \mathfrak{a}_{H}\right)$, respectively. We set

$$
\begin{aligned}
\mathfrak{n} & :=\bigoplus_{\left.\alpha\right|_{\mathfrak{a}_{H}} \in \Sigma^{+}\left(\mathfrak{g}, \mathfrak{a}_{H}\right)} \mathfrak{g}\left(\mathfrak{a}_{G} ; \alpha\right)=\bigoplus_{\lambda \in \Sigma^{+}\left(\mathfrak{g}, \mathfrak{a}_{H}\right)} \mathfrak{g}\left(\mathfrak{a}_{H} ; \lambda\right), \\
\mathfrak{n}_{G} & :=\bigoplus_{\alpha \in \Sigma^{+}\left(\mathfrak{g}, \mathfrak{a}_{G}\right)} \mathfrak{g}\left(\mathfrak{a}_{G} ; \alpha\right) .
\end{aligned}
$$

Clearly $\mathfrak{n} \subset \mathfrak{n}_{G}$. We remark that $\mathfrak{n}_{G}$ is not necessarily $\sigma$-stable, but $\mathfrak{n}$ is $\sigma$-stable. So we have a direct sum decomposition:

$$
\mathfrak{n}=\mathfrak{n}^{\sigma}+\mathfrak{n}^{-\sigma}
$$

We write $\Delta\left(\mathfrak{n}^{ \pm \sigma}\right)$ for the set of $\mathfrak{a}_{H^{-}}$weights in $\mathfrak{n}^{ \pm \sigma}$. Then we have

$$
\Sigma^{+}\left(\mathfrak{g}, \mathfrak{a}_{H}\right)=\Delta\left(\mathfrak{n}^{\sigma}\right) \cup \Delta\left(\mathfrak{n}^{-\sigma}\right)
$$

which is not disjoint in general. Let $P_{G}$ be the minimal parabolic subgroup of $G$ that normalizes $\mathfrak{n}_{G}, \overline{P_{G}}$ the opposite parabolic, and $Q$ and $\bar{Q}$ the parabolic subgroups of $G$ corresponding to $\Sigma^{+}\left(\mathfrak{g}, \mathfrak{a}_{H}\right)$ and $-\Sigma^{+}\left(\mathfrak{g}, \mathfrak{a}_{H}\right)$, respectively. Then $\mathfrak{p}_{H}:=\mathfrak{q} \cap \mathfrak{h}$ is a minimal parabolic subalgebra of $\mathfrak{h}$. We set

$$
\begin{aligned}
M_{G} & :=Z_{K}\left(\mathfrak{a}_{G}\right), \\
M_{H} & :=Z_{H \cap K}\left(\mathfrak{a}_{H}\right), \\
A_{H} & :=\exp \left(\mathfrak{a}_{H}\right), \\
L & :=Z_{G}\left(\mathfrak{a}_{H}\right), L_{H}:=Z_{H}\left(\mathfrak{a}_{H}\right)=M_{H} A_{H} .
\end{aligned}
$$

Then we have

$$
Q=L N=L \exp (\mathfrak{n}), \quad P_{H}=L_{H} N^{\sigma}=M_{H} A_{H} N^{\sigma}
$$

We note $P_{G} \subset Q \supset P_{H}$ and $Q \cap \overline{P_{G}}=L \cap \overline{P_{G}}$. 
In Introduction, we considered the following two properties:

(PP) $\quad P_{H}$ has an open orbit on the real flag variety $G / P_{G}$,

(BB) $B_{H}$ has an open orbit on the complex flag variety $G_{\mathbb{C}} / B_{G}$.

We note that the conditions $(\mathrm{PP})$ and $(\mathrm{BB})$ are independent of coverings or connectedness of the groups, and depend only on the pair of the Lie algebras $(\mathfrak{g}, \mathfrak{h})$.

In addition to the properties (PP) and (BB), we consider

(QP) $\quad P_{H}$ has an open orbit on the real generalized flag variety $G / \bar{Q}$.

Among the three properties, we have:

Lemma 3.1. Let $(\mathfrak{g}, \mathfrak{h})$ be a symmetric pair. Then we have

1) $(\mathrm{BB}) \Rightarrow(\mathrm{PP}) \Rightarrow(\mathrm{QP})$.

2) If $\operatorname{rank}_{\mathbb{R}} H=\operatorname{rank}_{\mathbb{R}} G$, then $(\mathrm{PP}) \Leftrightarrow(\mathrm{QP})$.

Proof. 1) The implication $(\mathrm{BB}) \Rightarrow(\mathrm{PP})$ follows from [13, Lemmas 4.2 and 5.3]. The implication $(\mathrm{PP}) \Rightarrow(\mathrm{QP})$ is obvious because $P_{G}$ is conjugate to $\bar{P}_{G}$ and $\bar{P}_{G} \subset \bar{Q}$.

2) If $\mathfrak{a}_{H}=\mathfrak{a}_{G}$, then $P_{G}$ coincides with $Q$. Thus (PP) is equivalent to (QP).

Remark 3.2. 1) We defined (PP) and (BB) without assuming that $(\mathfrak{g}, \mathfrak{h})$ is a symmetric pair, however, we can define $(\mathrm{QP})$ only for symmetric pairs $(\mathfrak{g}, \mathfrak{h})$.

2) The equivalence $(\mathrm{PP}) \Leftrightarrow(\mathrm{QP})$ holds also for any irreducible symmetric pair $(\mathfrak{g}, \mathfrak{h})$ with $\operatorname{rank}_{\mathbb{R}} \mathfrak{h} \geq 2$ (see Corollary 2.3).

\subsection{Criterion for (PP) and (QP)}

We are ready to state a necessary and sufficient condition for the property (PP), and that for $(\mathrm{QP})$ in terms of the adjoint action of $Z_{H}\left(\mathfrak{a}_{H}\right)=M_{H} A_{H}$ on $\mathfrak{n}^{-\sigma}$.

Theorem 3.3. The following two conditions are equivalent:

(i) $(\mathfrak{g}, \mathfrak{h})$ satisfies $(\mathrm{PP})$.

(ii) $\left(M_{H} \cap M_{G}\right) A_{H}$ has an open orbit on $\mathfrak{n}^{-\sigma}$ via the adjoint action.

Theorem 3.4. Let $(G, H)$ be a reductive symmetric pair. Then the following two conditions are equivalent:

(i) $(\mathfrak{g}, \mathfrak{h})$ satisfies $(\mathrm{QP})$. 
(ii) $Z_{H}\left(\mathfrak{a}_{H}\right)=M_{H} A_{H}$ has an open orbit on $\mathfrak{n}^{-\sigma}$.

For the proof of Theorems 3.3 and 3.4, we need a basic structural result on the centralizer of $\mathfrak{a}_{H}$ and $\mathfrak{a}_{G}$, respectively.

Lemma 3.5. 1) $Z_{\mathfrak{h} \cap \mathfrak{k}}\left(\mathfrak{a}_{H}\right) \cap Z_{\mathfrak{k}}\left(\mathfrak{a}_{G}\right)=Z_{\mathfrak{h} \cap \mathfrak{k}}\left(\mathfrak{a}_{G}\right)$.

2) $Z_{\mathfrak{h} \cap \mathfrak{k}}\left(\mathfrak{a}_{H}\right)+Z_{\mathfrak{k}}\left(\mathfrak{a}_{G}\right)=Z_{\mathfrak{k}}\left(\mathfrak{a}_{H}\right)$.

3) $Z_{\mathfrak{g}}\left(\mathfrak{a}_{H}\right)=Z_{\mathfrak{h} \cap \mathfrak{k}}\left(\mathfrak{a}_{H}\right)+\left(Z_{\mathfrak{g}}\left(\mathfrak{a}_{H}\right) \cap \overline{\mathfrak{p}}_{G}\right)$.

Proof. 1) Clear from $\mathfrak{a}_{H} \subset \mathfrak{a}_{G}$.

2) If $\left.\alpha\right|_{\mathfrak{a}_{H}}=0$ then $\sigma \theta \alpha=\alpha$, and therefore the involution $\sigma \theta$ stabilizes $\mathfrak{g}\left(\mathfrak{a}_{G} ; \alpha\right)$ with $\left.\alpha\right|_{\mathfrak{a}_{H}}=0$. Thus we have a direct sum decomposition

$$
\mathfrak{g}\left(\mathfrak{a}_{G} ; \alpha\right)=\mathfrak{g}^{\sigma \theta}\left(\mathfrak{a}_{G} ; \alpha\right)+\mathfrak{g}^{-\sigma \theta}\left(\mathfrak{a}_{G} ; \alpha\right) .
$$

We claim that $\mathfrak{g}^{-\sigma \theta}\left(\mathfrak{a}_{G} ; \alpha\right)=\{0\}$ for any $\alpha \in \Sigma\left(\mathfrak{g}, \mathfrak{a}_{G}\right)$ with $\left.\alpha\right|_{\mathfrak{a}_{H}}=0$. In fact, suppose that a nonzero element $X \in \mathfrak{g}\left(\mathfrak{a}_{G} ; \alpha\right)$ satisfies $\sigma \theta X=-X$. Then $X+\sigma X \neq 0$ because $\sigma X \in \mathfrak{g}\left(\mathfrak{a}_{G} ; \sigma \alpha\right)=\mathfrak{g}\left(\mathfrak{a}_{G} ;-\alpha\right)$ and $\mathfrak{g}\left(\mathfrak{a}_{G} ; \alpha\right) \cap \mathfrak{g}\left(\mathfrak{a}_{G} ;-\alpha\right)=\{0\}$ if $\alpha \neq 0$. On the other hand,

$$
X+\sigma X=X-\theta X \in \mathfrak{h}^{-\theta} .
$$

Since $\left[\mathfrak{a}_{H}, X+\sigma X\right]=\{0\}$, it contradicts the maximality of $\mathfrak{a}_{H}$ as an abelian subspace in $\mathfrak{h}^{-\theta}$. Thus we have shown the claim.

Therefore we have the following direct sum decomposition

$$
Z_{\mathfrak{g}}\left(\mathfrak{a}_{H}\right)=\bigoplus_{\left.\alpha\right|_{\mathfrak{a}_{H}}=0} \mathfrak{g}\left(\mathfrak{a}_{G} ; \alpha\right)=\mathfrak{g}\left(\mathfrak{a}_{G} ; 0\right) \oplus \bigoplus_{\substack{\left.\alpha\right|_{\mathfrak{a}_{H}}=0 \\ \alpha \neq 0}} \mathfrak{g}^{\sigma \theta}\left(\mathfrak{a}_{G} ; \alpha\right) .
$$

Taking the intersection with $\mathfrak{k}$, we get the identity

$$
\begin{aligned}
Z_{\mathfrak{k}}\left(\mathfrak{a}_{H}\right) & =\left(\mathfrak{g}\left(\mathfrak{a}_{G} ; 0\right) \cap \mathfrak{k}\right) \oplus \bigoplus_{\substack{\left.\alpha\right|_{\mathfrak{a}_{H}}=0, \alpha \neq 0}}\left(\mathfrak{g}^{\sigma \theta}\left(\mathfrak{a}_{G} ; \alpha\right) \cap \mathfrak{k}\right) \\
& =Z_{\mathfrak{k}}\left(\mathfrak{a}_{G}\right)+Z_{\mathfrak{h} \cap \mathfrak{k}}\left(\mathfrak{a}_{H}\right) .
\end{aligned}
$$

3) By the Iwasawa decomposition of the reductive subalgebra $Z_{\mathfrak{g}}\left(\mathfrak{a}_{H}\right)$, we have

$$
Z_{\mathfrak{g}}\left(\mathfrak{a}_{H}\right)=Z_{\mathfrak{k}}\left(\mathfrak{a}_{H}\right)+\left(Z_{\mathfrak{g}}\left(\mathfrak{a}_{H}\right) \cap \overline{\mathfrak{p}}_{G}\right) .
$$

Combining this with the second statement, we have

$$
Z_{\mathfrak{g}}\left(\mathfrak{a}_{H}\right)=Z_{\mathfrak{h} \cap \mathfrak{k}}\left(\mathfrak{a}_{H}\right)+Z_{\mathfrak{k}}\left(\mathfrak{a}_{G}\right)+\left(Z_{\mathfrak{g}}\left(\mathfrak{a}_{H}\right) \cap \overline{\mathfrak{p}}_{G}\right)=Z_{\mathfrak{h} \cap \mathfrak{k}}\left(\mathfrak{a}_{H}\right)+\left(Z_{\mathfrak{g}}\left(\mathfrak{a}_{H}\right) \cap \overline{\mathfrak{p}}_{G}\right)
$$

because $Z_{\mathfrak{k}}\left(\mathfrak{a}_{G}\right) \subset Z_{\mathfrak{g}}\left(\mathfrak{a}_{H}\right) \cap \overline{\mathfrak{p}}_{G}$. 
The following lemma is known ([2]), but we give a proof for the sake of completeness.

Lemma 3.6. Suppose $N$ is a simply connected nilpotent Lie group with an involutive automorphism $\sigma$.

1) The exponential map $\exp : \mathfrak{n} \rightarrow N$ induces bijections $\mathfrak{n}^{\sigma} \stackrel{\sim}{\rightarrow} N^{\sigma}$ and $\mathfrak{n}^{-\sigma} \stackrel{\sim}{\rightarrow} N^{-\sigma}$.

2) The following map is also bijective:

$$
\mathfrak{n}^{\sigma}+\mathfrak{n}^{-\sigma} \rightarrow N, \quad(X, Y) \mapsto \exp X \exp Y
$$

Proof. 1) Since $N$ is simply connected and nilpotent, the exponential map is bijective. We write $\log : N \rightarrow \mathfrak{n}$ for its inverse. Then, for the first statement, it is sufficient to prove the surjectivity of the restriction $\mathfrak{n}^{ \pm \sigma} \rightarrow N^{ \pm \sigma}$. Take an arbitrary $y \in N$ such that $\sigma(y)=y^{ \pm 1}$. Then $Y:=\log y$ satisfies $\exp (\sigma Y)=\exp ( \pm Y)$, whence $\sigma Y= \pm Y$. Thus exp : $\mathfrak{n}^{\sigma} \rightarrow N^{\sigma}$ and $\mathfrak{n}^{-\sigma} \rightarrow N^{-\sigma}$ are both surjective.

2) Clearly the map (3.1) is injective. To see (3.1) is surjective, we take $z \in N$. Since $z^{-1} \sigma(z) \in N^{-\sigma}$, we have $Y:=-\frac{1}{2} \log \left(z^{-1} \sigma(z)\right) \in \mathfrak{n}^{-\sigma}$. We set $x:=z \exp (-Y)$. Then $x \sigma(x)^{-1}=z \exp (-2 Y) \sigma(z)^{-1}=e$. Thus $X:=\log (x) \in \mathfrak{n}^{\sigma}$ and $z=\exp X \exp Y$. Hence we have shown that the map (3.1) is surjective, too.

Lemma 3.7. We let $Z_{H}\left(\mathfrak{a}_{H}\right)=M_{H} A_{H}$ act linearly on $\mathfrak{n}^{-\sigma}$. Then the natural inclusion $\mathfrak{n}^{-\sigma} \stackrel{\exp }{\rightarrow} N^{-\sigma} \hookrightarrow Q$ induces the following bijections:

$$
\begin{aligned}
\mathfrak{n}^{-\sigma} /\left(M_{H} \cap M_{G}\right) A_{H} & \stackrel{\sim}{\rightarrow} P_{H} \backslash Q /\left(L \cap \overline{P_{G}}\right), \\
\mathfrak{n}^{-\sigma} / M_{H} A_{H} & \stackrel{\sim}{\rightarrow} P_{H} \backslash Q / L .
\end{aligned}
$$

Proof. It follows from Lemmas 3.5 and 3.6 that

$$
Q=N L=N M_{H}\left(L \cap \overline{P_{G}}\right)=M_{H} N^{\sigma} \exp \left(\mathfrak{n}^{-\sigma}\right)\left(L \cap \overline{P_{G}}\right) .
$$

Thus the map (3.2) is surjective, and so is (3.3).

1) Suppose two elements $X_{1}, X_{2} \in \mathfrak{n}^{-\sigma}$ have the same image in (3.2). This means that there exist $l_{H} \in L_{H}=M_{H} A_{H}, n_{H} \in N^{\sigma}$, and $l \in L \cap \overline{P_{G}}$ such that $x_{i}=\exp \left(X_{i}\right)$ $(i=1,2)$ satisfy $x_{1}=l_{H} n_{H} x_{2} l$. Then we have

$$
L \ni l_{H}^{-1} l^{-1}=\left(l_{H}^{-1} x_{1}^{-1} l_{H}\right) n_{H} x_{2} \in N^{-\sigma} N^{\sigma} N^{-\sigma}=N
$$

and therefore $l=l_{H}^{-1}, n_{H}=e$, and $l_{H}^{-1} x_{1} l_{H}=x_{2}$. Hence $\operatorname{Ad}\left(l_{H}\right) X_{2}=X_{1}$. Since $l_{H}=l^{-1}$ belongs to

$$
M_{H} A_{H} \cap\left(L \cap \bar{P}_{G}\right)=\left(M_{H} \cap M_{G}\right) A_{H},
$$


the map (3.2) is injective.

2) The proof parallels to that for (3.2). The only difference is that $l \in L$ instead of the previous condition $l \in L \cap \bar{P}_{G}$, and thus $l_{H}=l^{-1}$ belongs to $M_{H} A_{H} \cap L=M_{H} A_{H}$. Hence $X_{1}$ and $X_{2}$ give the same equivalence class under the action of $M_{H} A_{H}$.

We are ready to complete the proof of Theorems 3.3 and 3.4 .

Proof of Theorem 3.3. Since any minimal parabolic subgroup is conjugate to each other by inner automorphisms, (PP) is equivalent to the existence of an open $P_{H}$-orbit in $G / \bar{P}_{G}$. By the Bruhat decomposition, the $Q$-orbit through the origin $o=e \bar{P}_{G}$ in $G / \bar{P}_{G}$ is open dense because $P_{G} \subset Q$. This open orbit is given by $Q /\left(Q \cap \bar{P}_{G}\right)=Q /\left(L \cap \bar{P}_{G}\right)$ as a homogeneous space of $Q$. Since $Q$ contains $P_{H}$, the condition (PP) is equivalent to the existence of an open $P_{H^{-}}$orbit in $Q /\left(L \cap \bar{P}_{G}\right)$. By Lemma 3.7, this amounts to the existence of an open $\left(M_{H} \cap M_{G}\right) A_{H}$ orbit in $\mathfrak{n}^{-\sigma}$.

Proof of Theorem 3.4. The proof is similar to that for Theorem 3.3. In fact, since the $Q$-orbit through the origin $o=e \bar{Q}$ in $G / \bar{Q}$ is open dense and given by $Q /(Q \cap \bar{Q})=Q / L$, the condition (QP) is equivalent to the existence of an open $P_{H^{-}}$orbit in $Q / L$, which in turn is equivalent to the existence of an open $M_{H} A_{H^{-}}$orbit in $\mathfrak{n}^{-\sigma}$ by Lemma 3.7.

\section{$3.3 \quad c$-dual of symmetric pairs and (QP)}

For a symmetric pair $(\mathfrak{g}, \mathfrak{h})$ defined by an involutive automorphism $\sigma$ of $\mathfrak{g}$, we write

$$
\mathfrak{g}=\mathfrak{g}^{\sigma}+\mathfrak{g}^{-\sigma}
$$

for the eigenspace decomposition of $\sigma$ with eigenvalues +1 and -1 as before. Then $\mathfrak{h}=\mathfrak{g}^{\sigma}$. We set

$$
\mathfrak{g}^{c}:=\mathfrak{g}^{\sigma}+\sqrt{-1} \mathfrak{g}^{-\sigma}
$$

Then the vector space $\mathfrak{g}^{c}$ carries a natural Lie algebra structure, and the pair $\left(\mathfrak{g}^{c}, \mathfrak{h}\right)$ forms a symmetric pair by the restriction of the complex linear extension of $\sigma$ to $\mathfrak{g}_{\mathbb{C}}=\mathfrak{g} \otimes_{\mathbb{R}} \mathbb{C}$. The pair $\left(\mathfrak{g}^{c}, \mathfrak{h}\right)$ is called the $c$-dual of the symmetric pair $(\mathfrak{g}, \mathfrak{h})$. We note that $\mathfrak{g}$ is reductive if and only if $\mathfrak{g}^{c}$ is reductive.

Example 3.8. 1) The $c$-dual of the "group case" $(\mathfrak{g} \oplus \mathfrak{g}, \operatorname{diag} \mathfrak{g})$ is isomorphic to the pair $\left(\mathfrak{g}_{\mathbb{C}}, \mathfrak{g}\right)$ where the involution of $\mathfrak{g}_{\mathbb{C}}$ is given by the complex conjugation with respect to the real form $\mathfrak{g}$.

2) The complex symmetric pair $\left(\mathfrak{g}_{\mathbb{C}}, \mathfrak{h}_{\mathbb{C}}\right)$ is self $c$-dual. 
Proposition 3.9. A reductive symmetric pair $(\mathfrak{g}, \mathfrak{h})$ satisfies (QP) if and only if the c-dual $\left(\mathfrak{g}^{c}, \mathfrak{h}\right)$ satisfies $(\mathrm{QP})$.

Proof. By the criterion in Theorem 3.4, the reductive symmetric pair $(\mathfrak{g}, \mathfrak{h})$ (respectively, the $c$-dual $\left(\mathfrak{g}^{c}, \mathfrak{h}\right)$ ) satisfies $(\mathrm{QP})$ if and only if the group $Z_{H}\left(\mathfrak{a}_{H}\right)$ has an open orbit in $\mathfrak{n}^{-\sigma}$ (respectively, in $\sqrt{-1} \mathfrak{n}^{-\sigma}$ ). Since $\mathfrak{n}^{-\sigma}$ and $\sqrt{-1} \mathfrak{n}^{-\sigma}$ are isomorphic to each other as modules of the group $Z_{H}\left(\mathfrak{a}_{H}\right)$, we get the proposition.

Remark 3.10. An analogous statement to Proposition 3.9 does not hold for (PP) in general.

\subsection{Further properties for (QP)}

In order to screen the symmetric pairs that do not satisfy (QP), it is convenient to find a necessary condition for (QP) in terms of the restricted root system.

Here is the one that we frequently use in later sections:

Proposition 3.11. If $(\mathfrak{g}, \mathfrak{h})$ satisfies (QP), then elements of $\Delta\left(\mathfrak{n}^{-\sigma}\right)$ are linearly independent. In particular, we have

$$
\operatorname{rank}_{\mathbb{R}} H \geq \# \Delta\left(\mathfrak{n}^{-\sigma}\right)
$$

where $\# \Delta\left(\mathfrak{n}^{-\sigma}\right)$ denotes the cardinality of the weights of $\mathfrak{a}_{H}$ in $\mathfrak{n}^{-\sigma}$ without counting the multiplicities.

The converse statement of Proposition 3.11 is not true; however, we shall see that the condition (3.4) is a fairly good criterion for (QP). For example if $(G, H)=$ $\left(S O^{*}(2 p+2 q), S O^{*}(2 p) \times S O^{*}(2 q)\right)$ then the condition (3.4) is equivalent to (QP) except for $(p, q)=(2,2)$, see Proposition 5.7.

For $\lambda \in \mathfrak{a}_{H}^{*}=\operatorname{Hom}_{\mathbb{R}}\left(\mathfrak{a}_{H}, \mathbb{R}\right)$, let $\chi_{\lambda}$ be the one-dimensional real representation of the abelian group $A_{H}$ given by

$$
\chi_{\lambda}(\exp Y)=\exp \langle\lambda, Y\rangle \quad \text { for } Y \in \mathfrak{a}_{H},
$$

and write $\mathbb{R}_{\lambda}(\simeq \mathbb{R})$ for the representation space of $\chi_{\lambda}$.

To prove the proposition, we need the following elementary lemma:

Lemma 3.12. Let $F$ be a finite subset of $\mathfrak{a}_{H}^{*}$. If $A_{H}$ has an open orbit in the vector space $\bigoplus_{\lambda \in F} \mathbb{R}_{\lambda}$, then $F$ consists of linearly independent elements. In particular, $\# F \leq$ $\operatorname{dim} \mathfrak{a}_{H}$. 
We return to the proof of Proposition 3.11:

Proof of Proposition 3.11. Since $\mathfrak{a}_{H}$ is a maximal abelian subspace in $\mathfrak{h}^{-\theta}$, we have $Z_{H}\left(\mathfrak{a}_{H}\right)=M_{H} A_{H}$ with $M_{H}=Z_{H \cap K}\left(\mathfrak{a}_{H}\right)$ compact. We equip $\mathfrak{n}^{-\sigma}$ with an $M_{H}$-inner product such that the decomposition

$$
\mathfrak{n}^{-\sigma} \simeq \bigoplus_{\lambda \in \Delta\left(\mathfrak{n}^{-\sigma}\right)} \mathfrak{g}^{-\sigma}\left(\mathfrak{a}_{H} ; \lambda\right)
$$

is orthogonal to each other.

Let $O_{\lambda}$ be the orthogonal group of the subspace $\mathfrak{g}^{-\sigma}\left(\mathfrak{a}_{H} ; \lambda\right)$. Then the quotient space of $\mathfrak{g}^{-\sigma}\left(\mathfrak{a}_{H} ; \lambda\right)$ by $O_{\lambda}$ is given by the "half line":

$$
\mathfrak{g}^{-\sigma}\left(\mathfrak{a}_{H} ; \lambda\right) / O_{\lambda} \simeq\left(\mathbb{R}_{\lambda}\right)_{\geq 0}
$$

Since the compact group $M_{H}$ preserves the inner product on $\mathfrak{n}^{-\sigma}$, we have a natural surjective map between the quotient spaces of $\mathfrak{n}^{-\sigma}$ :

$$
\mathfrak{n}^{-\sigma} / M_{H} \rightarrow \mathfrak{n}^{-\sigma} /\left(\prod_{\lambda \in \Delta\left(\mathfrak{n}^{-\sigma}\right)} O_{\lambda}\right) \simeq \prod_{\lambda \in \Delta\left(\mathfrak{n}^{-\sigma}\right)}\left(\mathfrak{g}^{-\sigma}\left(\mathfrak{a}_{H} ; \lambda\right) / O_{\lambda}\right) \simeq \prod_{\lambda \in \Delta\left(\mathfrak{n}^{-\sigma}\right)}\left(\mathbb{R}_{\lambda}\right)_{\geq 0}
$$

Then if $Z_{H \cap K}\left(\mathfrak{a}_{H}\right) A_{H}$ has an open orbit in $\mathfrak{n}^{-\sigma}$ via the adjoint representation, then $A_{H}$ has an open orbit in the quotient of $\mathfrak{n}^{-\sigma}$ by $M_{H}$. Therefore $A_{H}$ has an open orbit in

$$
\prod_{\lambda \in \Delta\left(\mathfrak{n}^{-\sigma}\right)}\left(\mathbb{R}_{\lambda}\right)_{\geq 0} \subset \bigoplus_{\lambda \in \Delta\left(\mathfrak{n}^{-\sigma}\right)} \mathbb{R}_{\lambda}
$$

too. Applying Lemma 3.12, we conclude that the elements of $\Delta\left(\mathfrak{n}^{-\sigma}\right)$ are linearly independent and therefore, $\# \Delta\left(\mathfrak{n}^{-\sigma}\right) \geq \operatorname{dim} \mathfrak{a}_{H}=\operatorname{rank}_{\mathbb{R}} H$. Hence the proposition is proved.

Next we analyze the inequality (3.4) in Proposition 3.11. For this, we denote by $W_{H}$ the Weyl group of the restricted root system $\Sigma\left(\mathfrak{h}, \mathfrak{a}_{H}\right)$. Then $W_{H}$ acts on the finite set $\Delta\left(\mathfrak{n}^{-\sigma}\right) \cup\left(-\Delta\left(\mathfrak{n}^{-\sigma}\right)\right)$, and consequently, we have an obvious inequality

$$
2 \# \Delta\left(\mathfrak{n}^{-\sigma}\right) \geq \#\left(W_{H} \cdot \lambda\right)
$$

for any $\lambda \in \Delta\left(\mathfrak{n}^{-\sigma}\right)$. Hence the inequality (3.4) implies that for any $\lambda \in \Delta\left(\mathfrak{n}^{-\sigma}\right)$, we have

$$
2 \operatorname{dim} \mathfrak{a}_{H} \geq \#\left(W_{H} \cdot \lambda\right)
$$

The inequality (3.5) gives strong constraints on both the root system $\Delta\left(\mathfrak{h}, \mathfrak{a}_{H}\right)$ and $\Delta\left(\mathfrak{n}^{-\sigma}\right)$. Let us examine (3.5) in an abstract setting (corresponding to the case where $\mathfrak{h}$ is simple) as follows: 
Lemma 3.13. Let $\Delta$ be an irreducible root system on a vector space $E$, and $W$ the Weyl group of $\Delta$. If there exists $\lambda \in E \backslash\{0\}$ such that

$$
2 \operatorname{dim} E \geq \#(W \cdot \lambda)
$$

then $\Delta$ is a classical root system. For an (irreducible) classical root system $\Delta$, we take a standard basis and the set $\Pi$ of simple roots as follows:

$$
\begin{aligned}
& \text { Case } 1: \Delta=A_{n}, \quad \Pi=\left\{\alpha_{i}=e_{i}-e_{i+1}: 1 \leq i \leq n\right\} \text { in } \mathbb{R}^{n} / \mathbb{R}\left(e_{1}+\cdots+e_{n+1}\right), \\
& \text { Case } \mathcal{L}: \Delta= \begin{cases}B_{n}, & \Pi=\left\{\alpha_{i}=e_{i}-e_{i+1}: 1 \leq i \leq n-1\right\} \cup\left\{\alpha_{n}=e_{n}\right\}, \\
C_{n}, & \Pi=\left\{\alpha_{i}=e_{i}-e_{i+1}: 1 \leq i \leq n-1\right\} \cup\left\{\alpha_{n}=2 e_{n}\right\}, \\
D_{n}, & \Pi=\left\{\alpha_{i}=e_{i}-e_{i+1}: 1 \leq i \leq n-1\right\} \cup\left\{\alpha_{n}=e_{n-1}+e_{n}\right\} .\end{cases}
\end{aligned}
$$

Here we assume $n \geq 1$ for $\Delta=A_{n}, n \geq 2$ for $\Delta=B_{n}, n \geq 3$ for $\Delta=C_{n}$, and $n \geq 4$ for $\Delta=D_{n}$.

Then $\lambda$ satisfying (3.6) must be of the following form:

$$
\begin{array}{lll}
\lambda \in \mathbb{R} e_{i} /\left(\mathbb{R}\left(e_{1}+\cdots+e_{n+1}\right)\right) & \text { for some } i(1 \leq i \leq n+1) & \text { in Case 1, } \\
\lambda \in \mathbb{R} e_{i} & \text { for some } i(1 \leq i \leq n) & \text { in Case 2. }
\end{array}
$$

Proof. For a root system $\Delta$, we consider the minimum cardinality of $W$-orbits defined by

$$
c(\Delta):=\inf _{\lambda \in E \backslash\{0\}} \#(W \cdot \lambda) .
$$

Let us compute $c(\Delta)$. For this, we fix a positive system $\Delta^{+}$, and write $\Pi=\left\{\alpha_{1}, \cdots, \alpha_{n}\right\}$ for the set of simple roots, and $\left\{\omega_{1}, \cdots, \omega_{n}\right\}$ for the set of fundamental weights. In order to compute the cardinality of the orbit $W \cdot \lambda$, we may assume $\lambda \in \overline{C_{+}} \backslash\{0\}$ without loss of generality, where $\overline{C_{+}}$is the dominant chamber defined by

$$
\overline{C_{+}}:=\left\{\sum_{i=1}^{n} a_{i} \omega_{i}: a_{1}, \cdots, a_{n} \geq 0\right\} .
$$

We define a partial order on $\overline{C_{+}}$by

$$
\lambda \succ \mu \quad \text { if } \lambda-\mu \in \overline{C_{+}} \text {. }
$$

We denote by $W_{\lambda}$ the isotropy subgroup of $W$ at $\lambda \in E$. Then $\#(W \cdot \lambda)=\# W / \# W_{\lambda}$. If $\lambda, \mu \in \overline{C_{+}}$satisfies $\lambda \succ \mu$, then there is an inclusion relation $W_{\lambda} \subset W_{\mu}$, and therefore $\# W \cdot \lambda \geq \# W \cdot \mu$. Thus $\# W \cdot \lambda$ attains its minimum only if $\lambda$ lies in the most singular part of the Weyl chamber, namely, only if $\lambda \in \mathbb{R}_{+} \omega_{i}(1 \leq i \leq n)$. In this case, 
$W_{\lambda}$ coincides with the Weyl group $W\left(\mathfrak{l}_{i}\right)$ of the Levi part $\mathfrak{l}_{i}$ of the maximal parabolic subgroup defined by the simple root $\alpha_{i}$. Thus we have

$$
c(\Delta)=\frac{\# W}{\max _{1 \leq i \leq n} \# W\left(\mathfrak{l}_{i}\right)} .
$$

This formula yields the explicit value of $c(\Delta)$ as in the table below, and also tells precisely when $\# W \cdot \lambda$ attains its minimum.

$\begin{array}{cccccccccc}\Delta & A_{n} & B_{n} & C_{n} & D_{n} & \mathfrak{e}_{6} & \mathfrak{e}_{7} & \mathfrak{e}_{8} & \mathfrak{f}_{4} & \mathfrak{g}_{2} \\ c(\Delta) & n+1 & 2 n & 2 n & 2 n & 27 & 56 & 240 & 24 & 6\end{array}$

Table 3.1: $c(\Delta)$ for simple root systems $\Delta$

For $\Delta=A_{n}$, we label simple roots as indicated. Then by a simple computation, we see that $\# W\left(\mathfrak{l}_{i}\right)$ attains its maximum $n$ ! at $i=1$ and $i=n$, and $\# W / \# W\left(\mathfrak{l}_{i}\right)>2 n$ for $2 \leq i \leq n-1$. Thus the inequality (3.6) holds for $\lambda \in \overline{C_{+}} \backslash\{0\}$ if and only if $\lambda \in \mathbb{R}_{+} \omega_{1}$ or $\mathbb{R}_{+} \omega_{n}$, namely, $\lambda \in \mathbb{R}_{+} e_{1}$ or $\lambda \in \mathbb{R}_{-} e_{n+1} \bmod \mathbb{R}\left(e_{1}+\cdots+e_{n+1}\right)$.

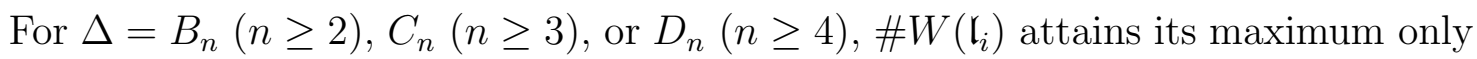
at $i=1$, and the inequality (3.6) is actually the equality when $\lambda \in \mathbb{R} \omega_{1}$.

For exceptional root systems $\Delta$, it is immediate from Table 3.1 that (3.6) does not hold. Hence Lemma 3.13 is proved.

We end this section with an easy-to-check necessary condition for (QP) when $\operatorname{rank}_{\mathbb{R}} G=\operatorname{rank}_{\mathbb{R}} H$. Proposition 3.14 below will be used in Section 8 when we deal with exceptional Lie algebras. For a real reductive Lie group $G$ with a Cartan involution $\theta$, we take a maximal abelian subspace $\mathfrak{a}_{G}$ in $\mathfrak{g}^{-\theta}$ and fix a positive system $\Sigma^{+}\left(\mathfrak{g}, \mathfrak{a}_{G}\right)$ as before. We set

$$
\begin{aligned}
m(G) & :=\max _{\alpha \in \Sigma\left(\mathfrak{g}, \mathfrak{a}_{G}\right)} \operatorname{dim}_{\mathbb{R}} \mathfrak{g}\left(\mathfrak{a}_{G} ; \alpha\right), \\
n(G) & :=\sum_{\alpha \in \Sigma^{+}\left(\mathfrak{g}, \mathfrak{a}_{G}\right)} \operatorname{dim}_{\mathbb{R}} \mathfrak{g}\left(\mathfrak{a}_{G} ; \alpha\right) .
\end{aligned}
$$

We note that $n(G)$ is equal to the dimension of the real flag variety $G / P_{G}$.

Proposition 3.14. Assume $\operatorname{rank}_{\mathbb{R}} G=\operatorname{rank}_{\mathbb{R}} H$. If the symmetric pair $(G, H)$ satisfies $(\mathrm{QP})$, then

$$
n(G)-n(H) \leq m(G) \operatorname{rank}_{\mathbb{R}} H .
$$


Proof. Since $\mathfrak{a}_{H}=\mathfrak{a}_{G}$ by the real rank assumption, we have

$$
m(G) \# \Delta\left(\mathfrak{n}^{-\sigma}\right) \geq \operatorname{dim} \mathfrak{n}^{-\sigma}=n(G)-n(H) .
$$

Hence the inequality (3.9) implies $\operatorname{rank}_{\mathbb{R}} H \geq \# \Delta\left(\mathfrak{n}^{-\sigma}\right)$. Thus Proposition 3.14 follows from Proposition 3.11 .

\section{Strong Gelfand pairs and their real forms}

This section focuses on (BB), which is much stronger than (PP) for real reductive pair $(\mathfrak{g}, \mathfrak{h})$ in general unless both $\mathfrak{g}$ and $\mathfrak{h}$ are quasi-split Lie algebras. We begin with the "complex case". In this case the condition (BB) is also referred to as a strong Gelfand pair.

Proposition 4.1. Suppose that $(\mathfrak{g}, \mathfrak{h})$ is a symmetric pair such that $\mathfrak{g}$ is a complex simple Lie algebra and $\mathfrak{h}$ is a complex subalgebra. Then the following three conditions are equivalent:

(i) The pair $(\mathfrak{g}, \mathfrak{h})$ satisfies $(\mathrm{PP})$.

(ii) The pair $(\mathfrak{g}, \mathfrak{h})$ satisfies (BB).

(iii) $(\mathfrak{g}, \mathfrak{h})$ is isomorphic to $(\mathfrak{s l}(n+1, \mathbb{C}), \mathfrak{g l}(n, \mathbb{C}))$ or $(\mathfrak{s o}(n+1, \mathbb{C}), \mathfrak{s o}(n, \mathbb{C}))$ up to outer automorphisms.

Proof. The equivalence (ii) $\Leftrightarrow$ (iii) was proved by Krämer [17]. Since any minimal parabolic subgroup is a Borel subgroup for complex reductive Lie groups, the equivalence (i) $\Leftrightarrow$ (ii) is obvious.

Alternatively, the proof of Proposition 4.1 is covered by special cases of our propositions in later sections:

Proposition $5.6 \quad(\mathfrak{o}(m+n, \mathbb{C}), \mathfrak{o}(m, \mathbb{C})+\mathfrak{o}(n, \mathbb{C}))$.

Proposition 6.2 $\operatorname{rank}_{\mathbb{R}} H=1$.

Proposition $7.2 \quad \operatorname{rank}_{\mathbb{R}} H \geq 2$ or $(\mathfrak{g}, \mathfrak{h}) \not(\mathfrak{o}(m+n, \mathbb{C}), \mathfrak{o}(m, \mathbb{C})+\mathfrak{o}(n, \mathbb{C}))$.

See also Propositions 5.4 and 5.12 for an alternative and direct proof for the pairs $(\mathfrak{s l}(m+n, \mathbb{C}), \mathfrak{s}(\mathfrak{g l}(m, \mathbb{C})+\mathfrak{g l}(n, \mathbb{C})))$ and $(\mathfrak{s p}(m+n, \mathbb{C}), \mathfrak{s p}(m, \mathbb{C})+\mathfrak{s p}(n, \mathbb{C}))$, respectively. 
Remark 4.2. In [3, 17], the pair $(\mathfrak{s o}(8, \mathbb{C}), \mathfrak{s p i n}(7, \mathbb{C}))$ also appears in the classification. However, it is isomorphic to the pair $(\mathfrak{s o}(8, \mathbb{C}), \mathfrak{s o}(7, \mathbb{C}))$ by an outer automorphism of $\mathfrak{s o}(8, \mathbb{C})$. The automorphism arises from the triality of $D_{4}$ (see also Lemma 5.15).

Since the condition (BB) is determined by the complexification of the pair $(\mathfrak{g}, \mathfrak{h})$, we have:

Proposition 4.3. Let $(\mathfrak{g}, \mathfrak{h})$ be an irreducible symmetric pair. Then the following two conditions are equivalent:

(i) $(\mathfrak{g}, \mathfrak{h})$ satisfies $(\mathrm{BB})$.

(ii) $(\mathfrak{g}, \mathfrak{h})$ is isomorphic to (F1)- (F5).

Remark 4.4. The "group case" $\left(\mathfrak{g}^{\prime}+\mathfrak{g}^{\prime}, \operatorname{diag} \mathfrak{g}^{\prime}\right)$ satisfying $(\mathrm{BB})$ is either $\mathfrak{g}^{\prime} \simeq \mathfrak{s} l(2, \mathbb{R})$ or $\mathfrak{g}^{\prime} \simeq \mathfrak{s l}(2, \mathbb{C})$ when $\mathfrak{g}^{\prime}$ is simple. They are included as special cases of (F2) and (F5):

$$
\begin{aligned}
(\mathfrak{s l}(2, \mathbb{R})+\mathfrak{s l}(2, \mathbb{R}), \operatorname{diag} \mathfrak{s l}(2, \mathbb{R})) & \approx(\mathfrak{o}(2,2), \mathfrak{o}(2,1)) \\
& \approx(\mathfrak{o}(2,1)+\mathfrak{o}(2,1), \operatorname{diag} \mathfrak{o}(2,1)) . \\
(\mathfrak{s l}(2, \mathbb{C})+\mathfrak{s l}(2, \mathbb{C}), \operatorname{diag} \mathfrak{s l}(2, \mathbb{C})) & \simeq(\mathfrak{s o}(4, \mathbb{C}), \mathfrak{s o}(3, \mathbb{C})) \\
& \simeq(\mathfrak{o}(3,1)+\mathfrak{o}(3,1), \operatorname{diag} \mathfrak{o}(3,1)) .
\end{aligned}
$$

\section{Some classical and exceptional cases}

In this section, we deal with some classical symmetric pairs $(G, H)$ in matrix forms and one exceptional symmetric pair. Classical symmetric spaces have parameters such as $i, j, k$ and $l$ in $(G, H)=(O(i+j, k+l), O(i, k) \times O(j, l))$. We determine for which parameters they satisfy (PP) or (QP) by using the criteria, Theorems 3.3 and 3.4. The cases we treat in Section 5 cover $(\mathrm{C})-(\mathrm{H})$ in Theorem 1.3 except for (E4) (see Step 4 in Section 2). In particular, the implication (ii) $\Rightarrow$ (i) in Theorem 2.1 is proved in this section except for (E4). The case (E4) will be treated in Section 6 together with other symmetric pairs $(\mathfrak{g}, \mathfrak{h})$ with $\operatorname{rank}_{\mathbb{R}} \mathfrak{h}=1$.

\section{$5.1(G, H)=(U(i+j, k+l ; \mathbb{F}), U(i, k ; \mathbb{F}) \times U(j, l ; \mathbb{F}))$}

The open orbit properties (BB), (PP), and (QP) do not change if we replace $(G, H)$ by their coverings, connected components, or the quotients $(G / Z, H / H \cap Z)$ by a central subgroup $Z$ of $G$. Thus, we shall treat the disconnected group $O(p, q)$ rather than the 
connected group $S O_{0}(p, q)$, and the reductive group $U(p, q)$ rather than the semisimple group $S U(p, q)$.

The goal of this subsection is to prove the following:

Proposition 5.1. Let $(G, H):=(U(i+j, k+l ; \mathbb{F}), U(i, k ; \mathbb{F}) \times U(j, l ; \mathbb{F}))$ with $\mathbb{F}=\mathbb{R}$, $\mathbb{C}$ or the quarternionic number field $\mathbb{H}$. Suppose that $l \leq \min \{i, j, k\}$.

1) The pair $(G, H)$ satisfies (QP) if and only if

$$
l=0 \quad \text { and } \quad \min (i, j, k)=1
$$

2) The pair $(G, H)$ satisfies $(\mathrm{PP})$ if and only if

$$
l=0 \quad \text { and } \min (j, k)=1 .
$$

In particular, Proposition 5.1 proves the implication (ii) $\Rightarrow$ (i) in Theorem 1.3 for (E1), (E2), (E3), (F4), (F5), and (H4).

In order to prove Proposition 5.1, we begin with the following:

Lemma 5.2. If $(G, H)$ satisfies $(\mathrm{QP})$, then $l=0$.

Proof. By Theorem 3.4, (QP) is equivalent to the existence of an open orbit of $M_{H} A_{H}$ on $\mathfrak{n}^{-\sigma}$. Then the idea of the proof of the lemma is to find a non-trivial $M_{H} A_{H^{-}}$ invariant function on $\mathfrak{n}^{-\sigma}$ for $l>0$. By a simple matrix computation, we have natural isomorphisms of groups and vector spaces:

$$
\begin{aligned}
M_{H} A_{H} & \simeq\left(\mathbb{F}^{\times}\right)^{\min (i, k)} \times U(|i-k|, \mathbb{F}) \times\left(\mathbb{F}^{\times}\right)^{l} \times U(j-l, \mathbb{F}), \\
\mathfrak{n}^{-\sigma} & \simeq M(i, l ; \mathbb{F}) \oplus M(j, \min (i, k) ; \mathbb{F}) .
\end{aligned}
$$

Via these identifications, the adjoint action of an element $(a, A, b, B) \in M_{H} A_{H}$ on the vector space $\mathfrak{n}^{-\sigma}$ is given as

$$
(X, Y) \mapsto \begin{cases}\left(\left(\begin{array}{ll}
a & \\
& A
\end{array}\right) X b^{-1},\left(\begin{array}{ll}
b & \\
B
\end{array}\right) Y a^{-1}\right) & \text { for } i \geq k, \\
\left(a X b^{-1},\left(\begin{array}{rr}
b & \\
B
\end{array}\right) Y a^{-1}\right) & \text { for } i \leq k .\end{cases}
$$

In the above formula, we have identified $b \in\left(\mathbb{F}^{\times}\right)^{l}$ with a diagonal matrix in $G L(l, \mathbb{F})$, and likewise for $a \in\left(\mathbb{F}^{\times}\right)^{\min (i, k)}$.

According to the partition $j=l+(j-l)$, we write $Y \in M(j, \min (i, k) ; \mathbb{F})$ as a block matrix $Y=\left(\begin{array}{c}Y^{\prime} \\ *\end{array}\right)$ with $Y^{\prime} \in M(l, \min (i, k) ; \mathbb{F})$. 
Consider the matrix $X Y^{\prime} \in M(i, \min (i, k) ; \mathbb{F})$. In view of the formula (5.1) of the $M_{H} A_{H^{-}}$-action on $\mathfrak{n}^{-\sigma}$, the $(1,1)$-component of $X Y^{\prime}$, to be denoted by $z$, is transformed as

$$
z \mapsto a_{1} z a_{1}^{-1},
$$

where $a_{1} \in \mathbb{F}^{\times}$is the first component of $a$. This formula means that the real algebraic function

$$
\psi: \mathfrak{n}^{-\sigma} \rightarrow \mathbb{R}, \quad(X, Y) \mapsto|z|^{2}
$$

is $M_{H} A_{H}$-invariant. Clearly, $\psi$ is non-zero if $l>0$. Hence $M_{H} A_{H}$ cannot have an open orbit in $\mathfrak{n}^{-\sigma}$ if $l>0$.

To complete the proof of Proposition 5.1 we need the following elementary lemma:

Lemma 5.3. Suppose $0 \leq p^{\prime} \leq p$ and $p, q \geq 1$. We let $S:=U(1, \mathbb{F})^{p^{\prime}} \times U\left(p-p^{\prime}, \mathbb{F}\right) \times$ $\left(\mathbb{F}^{\times}\right)^{q}$ act on $M(p, q ; \mathbb{F})$ by

$$
X \mapsto\left(\begin{array}{ll}
a & \\
& A
\end{array}\right) X b^{-1} \quad \text { for }(a, A, b) \in L \text { and } X \in M(p, q ; \mathbb{F}) .
$$

Then the group $S$ has an open orbit in $M(p, q ; \mathbb{F})$ if and only if $p=1$ or $(q=1$ and $\left.p^{\prime}=0\right)$.

Proof. First we observe that $\left(\mathbb{F}^{\times}\right)^{q}$ acts on the quotient space $U(p, \mathbb{F}) \backslash M(p, q ; \mathbb{F})$ from the right. This action has an open orbit if and only if $p=1$ or $q=1$ by the Gauss decomposition for $U(p, \mathbb{F}) \backslash M(p, q ; \mathbb{F})$. Hence the equivalence assertion of Lemma 5.3 is proved for $p^{\prime}=0$. What remains to prove is that there is no open orbit if $p^{\prime}>0$ and $q=1$, but this is obvious.

Proof of Proposition 5.1. 1) By Lemma 5.2, we may and do assume $l=0$. Then the group

$$
M_{H} A_{H} \simeq\left(\mathbb{F}^{\times}\right)^{\min (i, k)} \times U(|i-k|, \mathbb{F}) \times U(j, \mathbb{F}) \ni(a, A, B)
$$

acts on $\mathfrak{n}^{-\sigma} \simeq M(j, \min (i, k) ; \mathbb{F})$ by $Y \mapsto B Y a^{-1}$. We observe that the second factor $U(|i-k|, \mathbb{F})$ of $M_{H} A_{H}$ acts trivially on $\mathfrak{n}^{-\sigma}$. Then by the criterion in Theorem 3.4, the first statement follows as a special case of Lemma 5.3 with $p=j$ and $q=\min (i, k)$.

2) We need to prove that (PP) holds if $l=0$ and $\min (j, k)=1$, and fails if $l=0,=1$ and $\min (j, k)>1$. We shall use Theorem 3.3 and Lemma 5.3 .

Case 2-1. $k=1$. Then $\operatorname{rank}_{\mathbb{R}} H=\operatorname{rank}_{\mathbb{R}} G(=1)$, and therefore (QP) is equivalent to (PP) by Lemma 3.1 (2). Hence (PP) holds.

Case 2-2. $j=1$. We apply Lemma 5.3 with $p=1$ and $q=\min (i, k)$ to conclude that the action of $\left(M_{H} \cap M_{G}\right) A_{H}$ has an open orbit in $\mathfrak{n}^{-\sigma} \simeq \mathbb{F}^{\min (i, k)}$. 
Case 2-3. $i=1$. We apply Lemma 5.3 with $p=j, p^{\prime}=\min (j, k-1)$ and $q=1$ to conclude that the action of $\left(M_{H} \cap M_{G}\right) A_{H}$ does not have an open orbit if $j, k>1$.

Hence the proof of Proposition 5.1 is completed.

\section{$5.2(G, H)=(G L(p+q, \mathbb{F}), G L(p, \mathbb{F}) \times G L(q, \mathbb{F}))$}

Next we treat the symmetric pair $(G, H)=(G L(p+q, \mathbb{F}), G L(p, \mathbb{F}) \times G L(q, \mathbb{F}))$ for $\mathbb{F}=\mathbb{R}, \mathbb{C}$ and $\mathbb{H}$. Surprisingly, the property $(\mathrm{PP})$ behaves uniformly for all $\mathbb{F}=\mathbb{R}, \mathbb{C}$ and $\mathbb{H}$ for this pair. In contrast, that the property $(\mathrm{BB})$ behaves differently for $\mathbb{F}=\mathbb{H}$ (see Remark 5.5 below).

Proposition 5.4. Let $p, q \geq 1$ and

$$
(G, H)=(G L(p+q, \mathbb{F}), G L(p, \mathbb{F}) \times G L(q, \mathbb{F})), \quad \mathbb{F}=\mathbb{R}, \mathbb{C} \text { or } \mathbb{H} .
$$

Then the following three conditions are equivalent:

(i) The pair $(G, H)$ satisfies (QP).

(ii) The pair $(G, H)$ satisfies $(\mathrm{PP})$.

(iii) $\min (p, q)=1$.

Remark 5.5. For $\min (p, q)=1,(G, H)$ satisfies $(\mathrm{BB})$ if and only if $\mathbb{F}=\mathbb{R}$ or $\mathbb{C}$. In fact, for $\mathbb{F}=\mathbb{H}$, the complexified Lie algebra

$$
\left(\mathfrak{g}_{\mathbb{C}}, \mathfrak{h}_{\mathbb{C}}\right) \simeq(\mathfrak{g l}(2(p+q), \mathbb{C}), \mathfrak{g l}(2 p, \mathbb{C})+\mathfrak{g l}(2 q, \mathbb{C}))
$$

cannot be a strong Gelfand pair (see Proposition 4.1).

Proof of Proposition 5.4. Via the isomorphisms

$$
M_{H} A_{H} \simeq\left(\mathbb{F}^{\times}\right)^{p+q}, \quad \mathfrak{n}^{-\sigma} \simeq M(p, q ; \mathbb{F})
$$

the adjoint action of $M_{H} A_{H}$ on $\mathfrak{n}^{-\sigma}$ is given as the action of $\left(\mathbb{F}^{\times}\right)^{p} \times\left(\mathbb{F}^{\times}\right)^{q}$ on $M(p, q ; \mathbb{F})$ by the left and right multiplication.

If $p, q \geq 2$, then

$$
M(p, q ; \mathbb{F}) \rightarrow \mathbb{R}, \quad X \mapsto\left|X_{11} X_{22}\right|^{2} /\left|X_{12} X_{21}\right|^{2}
$$

is well-defined on an open dense subset of $M(p, q ; \mathbb{F})$ and is invariant by the action of $\left(\mathbb{F}^{\times}\right)^{p} \times\left(\mathbb{F}^{\times}\right)^{q}$, and thus there is no open orbit.

Conversely, if $q=1$, then clearly $\left(\mathbb{F}^{\times}\right)^{p}$ has an open orbit in $\mathbb{F}^{p}$, and so does $M_{H} A_{H}$ in $\mathfrak{n}^{-\sigma}$. Thus the equivalence (i) $\Leftrightarrow$ (iii) follows from Theorem 3.4.

Since $\operatorname{rank}_{\mathbb{R}} H=\operatorname{rank}_{\mathbb{R}} G$, the equivalence (i) $\Leftrightarrow$ (ii) holds. Hence Proposition is proved. 


\section{$5.3(G, H)=(O(m+n, \mathbb{C}), O(m, \mathbb{C}) \times O(n, \mathbb{C}))$}

The main goal of this section is to prove the following proposition. The equivalence (i) $\Leftrightarrow$ (ii) $\Leftrightarrow$ (iii) is a special case of Proposition 4.1. We shall use Proposition 5.6 in Proposition 7.2 .

Proposition 5.6. Let

$$
(G, H)=(O(m+n, \mathbb{C}), O(m, \mathbb{C}) \times O(n, \mathbb{C})) \text { with } m, n \geq 1 .
$$

Then the following four conditions are equivalent:

(i) The pair $(G, H)$ satisfies (QP).

(ii) The pair $(G, H)$ satisfies $(\mathrm{PP})$.

(iii) $m=1, n=1$ or $(m, n)=(2,2)$.

(iv) $\operatorname{rank}_{\mathbb{R}} H \geq \# \Delta\left(\mathfrak{n}^{-\sigma}\right)$.

Proof. The equivalence (iii) $\Leftrightarrow($ iv): From the table below, we see that $(\mathfrak{g}, \mathfrak{h})$ satisfies (iv) if and only if $m=1, n=1$, or $(m, n)=(1,2)$.

\begin{tabular}{llll}
\multicolumn{1}{c}{$m$} & \multicolumn{1}{c}{$n$} & $\operatorname{rank}_{\mathbb{R}} H$ & $\# \Delta\left(\mathfrak{n}^{-\sigma}\right)$ \\
$2 p+1$ & $2 q+1$ & $p+q$ & $2 p q+p+q$ \\
$2 p+1$ & $2 q$ & $p+q$ & $2 p q+q$ \\
$2 p$ & $2 q$ & $p+q$ & $2 p q$
\end{tabular}

\section{$5.4(G, H)=\left(O^{*}(2 p+2 q), O^{*}(2 p) \times O^{*}(2 q)\right)$}

The goal of this subsection is to prove the following:

Proposition 5.7. Let

$$
(G, H)=\left(O^{*}(2 p+2 q), O^{*}(2 p) \times O^{*}(2 q)\right) \text { with } p, q \geq 1 .
$$

The following three conditions are equivalent:

(i) The pair $(G, H)$ satisfies $(\mathrm{QP})$.

(ii) The pair $(G, H)$ satisfies $(\mathrm{PP})$.

(iii) $\min (p, q)=1$.

In particular, Proposition 5.7 shows the implication (ii) $\Rightarrow$ (i) in Theorem 1.3 for (H3).

In order to give a proof of the implication (i) $\Rightarrow$ (iii) in Proposition 5.7, we use Proposition 3.11. For this, we need: 
Lemma 5.8. $\operatorname{rank}_{\mathbb{R}} H \geq \# \Delta\left(\mathfrak{n}^{-\sigma}\right)$ if and only if $\min (p, q)=1$ or $(p, q)=(2,2)$.

Proof. Lemma 5.8 is an immediate consequence of the formulae:

$$
\operatorname{rank}_{\mathbb{R}} \mathfrak{a}_{H}=\left[\frac{p}{2}\right]+\left[\frac{q}{2}\right] \quad \text { and } \# \Delta\left(\mathfrak{n}^{-\sigma}\right)=\left[\frac{p q}{2}\right] .
$$

Proof of Proposition 5.7. The implication (ii) $\Rightarrow$ (i) holds in general by Lemma 3.1 (1). By Lemma 5.8 and Proposition 3.11, (QP) holds only if $\min (p, q)=1$ or $(p, q)=(2,2)$. What remains to prove is:

- (QP) fails if $(p, q)=(2,2)$.

- (PP) holds if $q=1$.

In view of the isomorphism of symmetric pairs:

$$
\left(\mathfrak{o}^{*}(8), \mathfrak{o}^{*}(4)+\mathfrak{o}^{*}(4)\right) \simeq(\mathfrak{o}(2,6), \mathfrak{o}(2,2)+\mathfrak{o}(4))
$$

the first claim is regarded as a special case of Proposition 5.1, which we have already proved.

To see the second claim, suppose $q=1$. Then we have the following natural isomorphisms of groups and vector spaces, respectively:

$$
\begin{aligned}
M_{H} A_{H} & \simeq \begin{cases}\left(\mathbb{H}^{\times}\right)^{\frac{p}{2}} \times \mathbb{T} & (p \text { :even }), \\
\left(\mathbb{H}^{\times}\right)^{\frac{p-1}{2}} \times \mathbb{T}^{2} & (p \text { :odd }),\end{cases} \\
\left(M_{H} \cap M_{G}\right) A_{H} & \simeq\left(\mathbb{H}^{\times}\right)^{\left[\frac{p}{2}\right]} \times \mathbb{T}, \\
\mathfrak{n}^{-\sigma} & \simeq \mathbb{H}^{\left[\frac{p}{2}\right]} .
\end{aligned}
$$

Via these isomorphisms, the adjoint action of the first factor of $\left(M_{H} \cap M_{G}\right) A_{H}$ on $\mathfrak{n}^{-\sigma}$ is given by the natural action of $\left(\mathbb{H}^{\times}\right)^{\left[\frac{p}{2}\right]}$ on $\mathbb{H}^{\left[\frac{p}{2}\right]}$, which has obviously an open dense orbit. By Theorem 3.3, we conclude that (PP) holds if $q=1$.

Hence the proof of Proposition 5.7 is completed.

\section{$5.5(G, H)$ is of type $\left(C_{n}, A_{n}\right)$}

In this subsection, we treat the reductive symmetric pairs $(\mathfrak{g}, \mathfrak{h})$ that have the following three properties: 
The root system $\Sigma\left(\mathfrak{g}, \mathfrak{a}_{H}\right)$ is of type $C_{n}$, The root system $\Sigma\left(\mathfrak{h}, \mathfrak{a}_{H}\right)$ is of type $A_{n}$, Either $m^{+}(\lambda)=0$ of $m^{-}(\lambda)=0$ for each $\lambda \in \Sigma\left(\mathfrak{g}, \mathfrak{a}_{H}\right)$.

Here we define

$$
m^{ \pm}(\lambda)=\operatorname{dim}_{\mathbb{R}} \mathfrak{g}^{ \pm \sigma}\left(\mathfrak{a}_{H} ; \lambda\right) .
$$

The main results of this subsection are Propositions 5.9 and 5.11 .

Proposition 5.9. Let $(G, H)$ be one of the following symmetric pairs:

$$
\begin{aligned}
& (U(n, n ; \mathbb{F}), G L(n, \mathbb{F})) \quad \mathbb{F}=\mathbb{C} \text { or } \mathbb{H}, \\
& (S p(n, \mathbb{R}), G L(n, \mathbb{R})), \\
& \left(O^{*}(4 n), G L(n, \mathbb{H})\right) .
\end{aligned}
$$

Then the following three conditions are equivalent:

(i) The pair $(G, H)$ satisfies (QP).

(ii) The pair $(G, H)$ satisfies (PP).

(iii) $n=1$.

To begin with, we observe the following:

Lemma 5.10. The three families of symmetric pairs $(\mathfrak{g}, \mathfrak{h})$ in Proposition 5.9 satisfy (5.2), (5.3), and (5.4).

Proof. We take the standard basis $\left\{e_{1}, \cdots, e_{n}\right\}$ of $\mathfrak{a}_{H}^{*}$ such that $\Sigma\left(\mathfrak{h}, \mathfrak{a}_{H}\right)=\left\{ \pm\left(e_{i}-e_{j}\right)\right.$ : $1 \leq i<j \leq n\}$. We set $d=\operatorname{dim}_{\mathbb{R}} \mathbb{F}$. Then the pair of multiplicities $\left(\begin{array}{l}m^{+}(\lambda) \\ m^{-}(\lambda)\end{array}\right)$ is given as follows: 


$$
\begin{aligned}
& \mathfrak{g} \quad \begin{array}{llll}
\mathfrak{h} & e_{i}-e_{j} & e_{i}+e_{j} & 2 e_{l}
\end{array} \\
& \mathfrak{u}(n, n ; \mathbb{F}) \quad \mathfrak{g l}(n, \mathbb{F}) \quad\left(\begin{array}{l}
d \\
0
\end{array}\right) \quad\left(\begin{array}{l}
0 \\
d
\end{array}\right) \quad\left(\begin{array}{c}
0 \\
d-1
\end{array}\right) \\
& \mathfrak{s p}(n, \mathbb{R}) \quad \mathfrak{g l}(n, \mathbb{R}) \quad\left(\begin{array}{l}
1 \\
0
\end{array}\right) \quad\left(\begin{array}{l}
0 \\
1
\end{array}\right) \quad\left(\begin{array}{l}
0 \\
1
\end{array}\right) \\
& \mathfrak{s o}^{*}(4 n) \quad \mathfrak{g l}(n, \mathbb{H}) \quad\left(\begin{array}{l}
4 \\
0
\end{array}\right) \quad\left(\begin{array}{l}
0 \\
4
\end{array}\right) \quad\left(\begin{array}{l}
0 \\
1
\end{array}\right)
\end{aligned}
$$

Table 5.1: $\left(m^{+}(\lambda), m^{-}(\lambda)\right)$ for symmetric pairs of type $\left(C_{n}, A_{n}\right)$

The lemma is clear from Table 5.1.

Proof of Proposition 5.9. By (5.2), (5.3) and (5.4), $\# \Delta\left(\mathfrak{n}^{-\sigma}\right)$ is equal to half the difference of the cardinalities of roots in $C_{n}$ and $A_{n}$, namely,

$$
\# \Delta\left(\mathfrak{n}^{-\sigma}\right)=\frac{1}{2}\left(2 n^{2}-\left(n^{2}-n\right)\right)=\frac{1}{2} n(n+1) .
$$

Therefore, the inequality $\operatorname{rank}_{\mathbb{R}} H \geq \# \Delta\left(\mathfrak{n}^{-\sigma}\right)$ amounts to $n \geq \frac{1}{2} n(n+1)$, namely, $n=1$. By Proposition 3.11, we have shown the implication (i) $\Rightarrow$ (iii).

The equivalence (i) $\Leftrightarrow$ (ii) follows from Lemma 3.1 (2) because $\operatorname{rank}_{\mathbb{R}} H=\operatorname{rank}_{\mathbb{R}} G$.

Finally, the implication (iii) $\Rightarrow$ (i) is included in a special (and easy) case of other families, which we have already shown to satisfy (PP). In fact,

$$
\begin{aligned}
(\mathfrak{u}(1,1), \mathfrak{g l}(1, \mathbb{C})) & \simeq(\mathfrak{o}(2,1), \mathfrak{o}(1,1))+(\mathbb{R}, \mathbb{R}), \\
(\mathfrak{s p}(1,1), \mathfrak{g l}(1, \mathbb{H})) & \simeq(\mathfrak{o}(1,4), \mathfrak{o}(1,1)+\mathfrak{o}(3)), \\
(\mathfrak{s p}(1, \mathbb{R}), \mathfrak{g l}(1, \mathbb{R})) & \simeq(\mathfrak{o}(2,1), \mathfrak{o}(1,1)), \\
\left(\mathfrak{o}^{*}(4), \mathfrak{g l}(1, \mathbb{H})\right) & \simeq(\mathfrak{o}(3), \mathfrak{o}(3)) \oplus(\mathfrak{o}(1,2), \mathfrak{o}(1,1)) .
\end{aligned}
$$

We know that the symmetric pairs in the right-hand side satisfy (PP) as special cases of Proposition 5.1. Thus we have proved Proposition 5.9.

We end this subsection with the symmetric pair $(U(n, n ; \mathbb{F}), G L(n, \mathbb{F}))$ for $\mathbb{F}=\mathbb{R}$, which was excluded from Proposition 5.9 as a "degenerate case".

Proposition 5.11. Let $(G, H)=(O(n, n), G L(n, \mathbb{R}))(n \geq 2)$. Then $(\mathrm{QP}) \Leftrightarrow(\mathrm{PP}) \Leftrightarrow$ $n=2$ or 3 . 
Proof. The root multiplicities are given in the first row of Table 5.1. We observe that the long roots $\pm 2 e_{l}$ do not appear because $d=1$ for $\mathbb{F}=\mathbb{R}$. As a result, we have $\# \Delta\left(\mathfrak{n}^{-\sigma}\right)=\frac{1}{2} n(n-1)$, and the inequality $\operatorname{rank}_{\mathbb{R}} H \geq \# \Delta\left(\mathfrak{n}^{-\sigma}\right)$ amounts to $n \geq \frac{1}{2} n(n-1)$, namely, $n=2$ or 3 . Thus we have proved the implications $(\mathrm{PP}) \Leftrightarrow(\mathrm{QP})$ $\Rightarrow n=2$ or 3 by Proposition 3.11.

Conversely, for $n=2,3$, we observe the following isomorphisms:

$$
\begin{aligned}
& (\mathfrak{o}(2,2), \mathfrak{g l}(2, \mathbb{R})) \simeq(\mathfrak{o}(1,2), \mathfrak{o}(1,2)) \oplus(\mathfrak{o}(1,2), \mathfrak{o}(1,1)), \\
& (\mathfrak{o}(3,3), \mathfrak{g l}(3, \mathbb{R})) \simeq(\mathfrak{s} l(4, \mathbb{R}), \mathfrak{g l}(3, \mathbb{R})) .
\end{aligned}
$$

They satisfy (PP) as special cases of Propositions 5.1 and 4.3, respectively.

$$
\text { 5.6 }(G, H)=(S p(p+q, \mathbb{F}), S p(p, \mathbb{F}) \times S p(q, \mathbb{F})), \mathbb{F}=\mathbb{R} \text { or } \mathbb{C}
$$

Proposition 5.12. Let $p, q \geq 1$ and

$$
(G, H)=(S p(p+q, \mathbb{F}), S p(p, \mathbb{F}) \times S p(q, \mathbb{F})), \quad \mathbb{F}=\mathbb{R} \text { or } \mathbb{C} .
$$

Then $(\mathrm{QP}) \Leftrightarrow(\mathrm{PP}) \Leftrightarrow(p, q)=(1,1)$.

Proof. Take the standard basis $\left\{f_{1}, \cdots, f_{p+q}\right\}$ of $\mathfrak{a}_{H}^{*}=\mathfrak{a}_{G}^{*}$ such that

$$
\Delta\left(\mathfrak{n}^{-\sigma}\right)=\left\{f_{i} \pm f_{j}: 1 \leq i \leq p, p+1 \leq j \leq p+q\right\} .
$$

Then the inequality $\operatorname{rank}_{\mathbb{R}} H \geq \# \Delta\left(\mathfrak{n}^{-\sigma}\right)$ amounts to $p+q \geq 2 p q$, which holds only if $(p, q)=(1,1)$. Therefore, if $(G, H)$ satisfies $(\mathrm{QP})$, then $(p, q)=(1,1)$ by Theorem 3.4 and Proposition 3.11. Conversely, if $(p, q)=(1,1)$, then

$$
\begin{aligned}
& (\mathfrak{s p}(2, \mathbb{R}), \mathfrak{s p}(1, \mathbb{R})+\mathfrak{s p}(1, \mathbb{R})) \simeq(\mathfrak{o}(3,2), \mathfrak{o}(2,2)), \\
& (\mathfrak{s p}(2, \mathbb{C}), \mathfrak{s p}(1, \mathbb{C})+\mathfrak{s p}(1, \mathbb{C})) \simeq(\mathfrak{o}(5, \mathbb{C}), \mathfrak{o}(4, \mathbb{C})),
\end{aligned}
$$

which satisfy (PP) as we have seen in Propositions 5.1 and 5.6, respectively. Hence Proposition 5.12 is proved.

$$
5.7(G, H)=\left(O^{*}(2 p+2 q), U(p, q)\right)
$$

As a final example of classical symmetric pairs, we consider $(\mathfrak{g}, \mathfrak{h})=\left(\mathfrak{o}^{*}(2 p+2 q), \mathfrak{u}(p, q)\right)$ which is the $c$-dual of the symmetric pair $(\mathfrak{o}(2 p+2 q), \mathfrak{u}(p, q))$.

Proposition 5.13. Let

$$
(G, H)=\left(O^{*}(2 p+2 q), U(p, q)\right) \text { with } p \geq q \geq 1 .
$$


1) The pair $(G, H)$ satisfies (QP) if and only if $q=1$.

2) The pair $(G, H)$ satisfies $(\mathrm{PP})$ if and only if $(p, q)=(3,1),(2,1)$ or $(1,1)$.

Proof. We take the standard basis $\left\{e_{1}, \cdots, e_{q}\right\}$ of $\mathfrak{a}_{H}^{*}$ such that

$$
\Sigma\left(\mathfrak{g}, \mathfrak{a}_{H}\right) \subset\left\{ \pm e_{i} \pm e_{j}: 1 \leq i<j \leq q\right\} \cup\left\{ \pm e_{l}, \pm 2 e_{l}: 1 \leq l \leq q\right\}
$$

The inclusion is actually the equality if and only if $p>q$. Further, the root multiplicities $m^{ \pm}(\lambda)$ are given according to the parity of $p+q$ as follows:

Case 1. $p \equiv q \bmod 2$.

$\begin{array}{cccc}\lambda & \pm e_{i} \pm e_{j} & \pm e_{l} & \pm 2 e_{l} \\ m^{+}(\lambda) & 2 & 2(p-q) & 1 \\ m^{-}(\lambda) & 2 & 2(p-q) & 0\end{array}$

Case 2. $p \equiv q+1 \bmod 2$.

$\begin{array}{cccc}\lambda & \pm e_{i} \pm e_{j} & \pm e_{l} & \pm 2 e_{l} \\ m^{+}(\lambda) & 2 & 2(p-q+1) & 1 \\ m^{-}(\lambda) & 2 & 2(p-q+1) & 0\end{array}$

Thus we can take a positive system such that

$$
\Delta\left(\mathfrak{n}^{-\sigma}\right)=\left\{ \pm e_{i} \pm e_{j}: 1 \leq i<j \leq q\right\}\left(\cup\left\{ \pm e_{l}: 1 \leq l \leq q\right\} \text { for } p>q\right) .
$$

Hence the inequality $\operatorname{rank}_{\mathbb{R}} H \geq \# \Delta\left(\mathfrak{n}^{-\sigma}\right)$ implies

$$
q \geq \begin{cases}q(q-1) & \text { for } p=q \\ q(q-1)+q & \text { for } p>q\end{cases}
$$

By Proposition 3.11, if $(G, H)$ satisfies (QP) then $(p, q)=(2,2)$ or $q=1$.

Conversely, suppose that $(p, q)=(2,2)$. In view of the isomorphism

$$
\left(\mathfrak{o}^{*}(8), \mathfrak{u}(2,2)\right) \simeq(\mathfrak{o}(6,2), \mathfrak{o}(4,2)+\mathfrak{o}(2)),
$$

we see that $(G, H)$ does not satisfy (QP) by Proposition 5.1 (1).

Suppose now that $q=1$. Then $\operatorname{rank}_{\mathbb{R}} H=1$, and we shall show in Proposition 6.2 that $(G, H)$ satisfies (QP) for any $p$ and (PP) for $p \leq 3$ (see III in Table 6.2). This completes the proof of Proposition 5.13. 


\section{$\mathbf{5 . 8}(\mathfrak{g}, \mathfrak{h})=\left(\mathfrak{e}_{6(-26)}, \mathfrak{s o}(9,1)+\mathbb{R}\right)$}

The exceptional real Lie algebra $\mathfrak{g}:=\mathfrak{e}_{6(-26)}$ is a simple Lie algebra with the following property:

$$
\mathfrak{k} \simeq \mathfrak{f}_{4(-52)} \text { and } \operatorname{rank}_{\mathbb{R}} \mathfrak{g}=2 .
$$

The goal of this subsection is to prove the following:

Proposition 5.14. Let $(G, H)$ be a symmetric pair with Lie algebras

$$
(\mathfrak{g}, \mathfrak{h})=\left(\mathfrak{e}_{6(-26)}, \mathfrak{s o}(9,1)+\mathbb{R}\right)
$$

Then $(G, H)$ satisfies $(\mathrm{PP})$ and $(\mathrm{QP})$.

We begin with the Lie algebra $\mathfrak{g}=\mathfrak{e}_{6(-26)}$. Then the Lie algebra $\mathfrak{m}_{G}=Z_{\mathfrak{k}}\left(\mathfrak{a}_{G}\right) \simeq$ $\mathfrak{s o}(8)$ acts on $\mathfrak{n} \simeq \mathbb{R}^{24}$ via the adjoint action as the direct sum of the following three non-isomorphic 8-dimensional irreducible representations:

$$
\begin{array}{ll}
\text { Natural representation } i & \begin{array}{l}
\text { highest weight } \\
\lambda_{1}
\end{array}=(1,0,0,0), \\
\text { Half spin representation } \operatorname{spin}^{+} & \lambda_{2}=\frac{1}{2}(1,1,1,1), \\
\text { Half spin representation } \operatorname{spin}^{-} & \lambda_{3}=\frac{1}{2}(1,1,1,-1) .
\end{array}
$$

Here the highest weights are expressed by means of the standard basis of $D_{4}$ as in the proof of Lemma 3.13 .

These representations are the differentials of the representations of $\operatorname{Spin}(8)$, to be denoted by the same letters $i, \operatorname{spin}^{+}$, and $\operatorname{spin}^{-}$, respectively, which in turn induce three actions on the 7 -dimensional sphere $S^{7} \simeq\left(\mathbb{R}^{8}-\{0\}\right) / \mathbb{R}_{>0}$. We need the following:

Lemma 5.15. Let Spin(8) act diagonally on the direct product manifold $S^{7} \times S^{7}$ via any choice of two distinct 8-dimensional representations among $i$, $\operatorname{spin}^{+}$, and $\mathrm{spin}^{-}$. Then the action is transitive.

Proof. The automorphism of the Dynkin diagram $D_{4}$ gives rise to the triality in $\operatorname{Spin}(8)$. We denote by $\sigma$ the outer automorphism of $\operatorname{Spin}(8)$ of order three corresponding to the outer automorphism of $D_{4}$ as described in the figure below. 


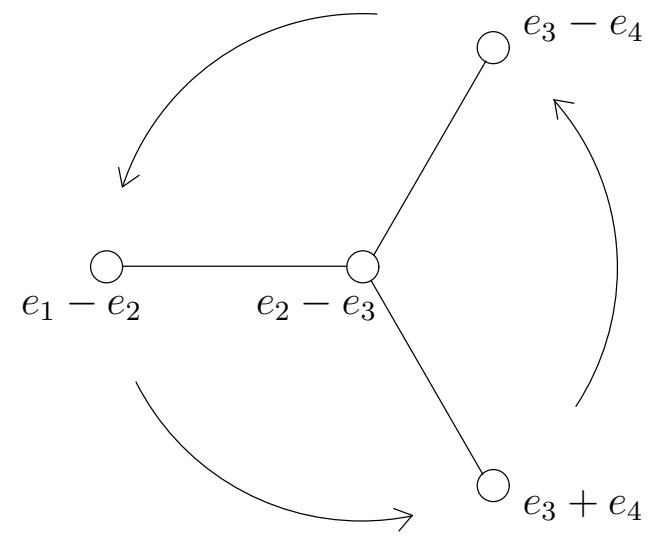

Then $\sigma$ induces the permutation of the set $\left\{\lambda_{1}, \lambda_{2}, \lambda_{3}\right\}$, by $\lambda_{1} \mapsto \lambda_{2} \mapsto \lambda_{3} \mapsto \lambda_{1}$, and thus the representations $i$, $\operatorname{spin}^{+}$, and spin $^{-}$are mutually equivalent by the outer automorphism group of $\operatorname{Spin}(8)$ (triliality of $D_{4}$ ). Hence, without loss of generality, we may and do assume that $\operatorname{Spin}(8)$ acts on $S^{7} \times S^{7}$ via $i \oplus \operatorname{spin}^{+}$.

First, we consider the action of $\operatorname{Spin}(8)$ on the first factor $S^{7}$ via the natural representation $\operatorname{Spin}(8) \stackrel{i}{\rightarrow} S O(8)$, which is a transitive action and gives rise to a natural diffeomorphism $\operatorname{Spin}(8) / \operatorname{Spin}(7) \simeq S^{7}$.

Second, we consider the action of the isotropy subgroup $\operatorname{Spin}(7)$ on the second factor $S^{7}$ via the following composition:

$$
\operatorname{Spin}(7) \hookrightarrow \operatorname{Spin}(8) \stackrel{\mathrm{spin}^{+}}{\rightarrow} S O(8) .
$$

This action is again transitive, and giving a natural diffeomorphism $\operatorname{Spin}(7) / G_{2} \simeq S^{7}$. Thus we have shown that $\operatorname{Spin}(8)$ acts transitively on $S^{7} \times S^{7}$ via $i \oplus \operatorname{spin}^{+}$.

For the Lie algebra $\mathfrak{h}=\mathfrak{o}(9,1)+\mathbb{R}$, the adjoint action of the Lie algebra $\mathfrak{m}_{H}$ on $\mathfrak{n}^{\sigma}=\mathfrak{n} \cap \mathfrak{h}$ is isomorphic to the natural representation of $\mathfrak{s o}(8)$ on $\mathbb{R}^{8}$.

We are ready to complete the proof of Proposition 5.14.

Proof of Proposition 5.14. Since $\operatorname{rank}_{\mathbb{R}} H=\operatorname{rank}_{\mathbb{R}} G(=2),(\mathrm{PP})$ is equivalent to (QP) by Lemma 3.1 .

The identity component $\left(M_{H}\right)_{0}$ of $M_{H}$ is isomorphic to $\operatorname{Spin}(8)$, and the adjoint action of $\left(M_{H}\right)_{0}$ on $\mathfrak{n}^{-\sigma} \simeq \mathfrak{n} / \mathfrak{n}^{\sigma}$ is isomorphic to the spin representation $\operatorname{spin}^{+} \oplus \operatorname{spin}^{-}$ of $\operatorname{Spin}(8)$ on $\mathbb{R}^{16}=\mathbb{R}^{8} \oplus \mathbb{R}^{8}$. Thus it induces a transitive action of $\operatorname{Spin}(8)$ on $S^{7} \times S^{7}$ by Lemma 5.15. On the other hand, since there are two distinct weights of $\mathfrak{a}_{H}$ on $\mathfrak{n}^{-\sigma}$, we conclude that the adjoint action of $M_{H} A_{H}$ has an open dense orbit in $\mathfrak{n}^{-\sigma} \simeq \mathbb{R}^{16}$. By Theorem 3.4, $(G, H)$ satisfies (QP). 


\section{Symmetric pair $(G, H)$ with $\operatorname{rank}_{\mathbb{R}} H=1$}

Since a minimal parabolic subgroup of a compact Lie group $K$ is $K$ itself, the following proposition is obvious by the Iwasawa decomposition $G=K A_{G} N=K P_{G}$ :

Proposition 6.1. Any Riemannian symmetric pair $(G, K)$ satisfies $(\mathrm{PP})$ and $(\mathrm{QP})$.

Among reductive symmetric pairs $(G, H)$, the Riemannian symmetric pair is characterized by the condition $\operatorname{rank}_{\mathbb{R}} H=0$. In this section, as the "next case" of Proposition 6.1, we highlight the case where $\operatorname{rank}_{\mathbb{R}} H=1$ and we give a classification of $(\mathfrak{g}, \mathfrak{h})$ satisfying (PP), see (E1)-(E4), (G2), or (H1), in Theorem 1.3.

In Table 6.1, we give a list of all irreducible symmetric pairs $(\mathfrak{g}, \mathfrak{h})$ with $\operatorname{rank}_{\mathbb{R}} \mathfrak{h}=$ 1. Since $(\mathfrak{g}, \mathfrak{h})$ and its $c$-dual $\left(\mathfrak{g}^{c}, \mathfrak{h}\right)$ have the same root multiplicities $m^{ \pm}(\lambda)=$ $\operatorname{dim} \mathfrak{g}^{ \pm \sigma}\left(\mathfrak{a}_{H} ; \lambda\right)$, we write them in the same row. Some of the symmetric pairs are labelled as $\mathrm{I}_{\mathbb{R}}, \mathrm{I}_{\mathbb{R}}^{c}, \cdots$, for which we give more detailed data in Table 6.2. We are now ready to state the main result of this section. This completes the proof of Theorems 1.3 and 2.2 for the classification of $(\mathfrak{g}, \mathfrak{h})$ with $(\mathrm{PP})$ and $(\mathrm{QP})$, respectively, under the assumption that $\operatorname{rank}_{\mathbb{R}} H=1$.

Proposition 6.2. Suppose $(G, H)$ is an irreducible symmetric pair with $\operatorname{rank}_{\mathbb{R}} H=1$.

1) The following two conditions (i) and (ii) on the pair $(G, H)$ are equivalent:

(i) The pair $(G, H)$ satisfies (QP).

(ii) The pair $(\mathfrak{g}, \mathfrak{h})$ is one of $\mathrm{I}_{\mathbb{F}}, \mathrm{I}_{\mathbb{F}}^{c}(\mathbb{F}=\mathbb{R}, \mathbb{C}, \mathbb{H}$, or $\mathbb{O})$, II, II' , III or $\mathrm{III}^{c}$.

2) The following two conditions (iii) and (iv) of the pair $(G, H)$ are equivalent:

(iii) The pair $(G, H)$ satisfies $(\mathrm{PP})$.

(iv) The pair $(\mathfrak{g}, \mathfrak{h})$ is one of $\mathrm{I}_{\mathbb{R}}^{c}, \mathrm{I}_{\mathbb{C}}^{c}, \mathrm{I}_{\mathbb{H}}^{c}, \mathrm{I}_{\mathbb{O}}^{c}, \mathrm{II}^{c}, \mathrm{III}^{c}, \mathrm{I}_{\mathbb{F}}(\mathbb{F}=\mathbb{R}, \mathbb{C}, \mathbb{H})$ with $p=0$ or $q=1$, II with $m=1,2$, or III with $m=1,2$.

Proof. We divide the proof into three steps.

Step 1. For the implication (i) $\Rightarrow$ (ii), we apply Proposition 3.11. Since $\operatorname{rank}_{\mathbb{R}} H=1$, if $(G, H)$ satisfies $(\mathrm{QP})$, then $m^{-}(\lambda)+m^{-}(2 \lambda) \leq 1$ by (3.4). In light of Table 6.1, we have shown that (i) implies (ii).

Step 2. In order to prove the equivalence (iii) $\Leftrightarrow$ (iv), it is sufficient to deal with symmetric pairs $(G, H)$ satisfying (QP) because (PP) implies (QP) (see Lemma 3.1). In particular, we may assume that $(G, H)$ satisfies (ii) by Step 1 . For the pairs $(\mathfrak{g}, \mathfrak{h})$ satisfying (ii), we give a list of the vector spaces $\mathfrak{n}^{-\sigma}$ on which the subalgebras $\left(\mathfrak{m}_{H} \cap\right.$ 
$\left.\mathfrak{m}_{G}\right)+\mathfrak{a}_{H} \subset \mathfrak{m}_{H}+\mathfrak{a}_{H}$ act via the adjoint representation in Table 6.2 . For $\mathrm{I}_{\mathbb{F}}(\mathbb{F}=\mathbb{R}, \mathbb{C}$, or $\mathbb{H})$ in this table, the action of $\mathfrak{u}(|p-q| ; \mathbb{F})$ on $\mathbb{F}^{q}$ is trivial if $p \geq q$ and is the natural action on the first factor of the decomposition $\mathbb{F}^{q}=\mathbb{F}^{q-p} \oplus \mathbb{F}^{p}$ if $q \geq p$. In view of this table, we see that $\left(M_{H} \cap M_{G}\right) A_{H}$ has an open orbit in $\mathfrak{n}^{-\sigma}$ if and only if (iv) holds. Hence the equivalence (iii) $\Leftrightarrow$ (iv) follows from Theorem 3.3 .

Step 3. The converse implication (i) $\Leftarrow$ (ii) follows from Step 2 and Proposition 3.9 because $\mathrm{I}_{\mathbb{F}}^{c}$, $\mathrm{II}^{c}$, and $\mathrm{III}^{c}$ are the $c$-duals of $\mathrm{I}_{\mathbb{F}}$, II, and III, respectively. 
Table 6.1: Irreducible symmetric pairs $(\mathfrak{g}, \mathfrak{h})$ with $\operatorname{rank}_{\mathbb{R}} \mathfrak{h}=1$

\begin{tabular}{|c|c|c|c|}
\hline & $\begin{array}{l}\mathfrak{g} \\
\mathfrak{g}^{c}\end{array}$ & $\mathfrak{h}$ & $\left(\begin{array}{ll}m^{+}(\lambda) & m^{+}(2 \lambda) \\
m^{-}(\lambda) & m^{-}(2 \lambda)\end{array}\right)$ \\
\hline $\begin{array}{l}\mathrm{I}_{\mathbb{R}} \\
\mathrm{I}_{\mathbb{R}}^{c}\end{array}$ & $\begin{array}{l}\mathfrak{s o}(p+1, q+1) \\
\mathfrak{s o}(p+q+1,1)\end{array}$ & $\mathfrak{s o}(q)+\mathfrak{s o}(p+1,1)$ & $\left(\begin{array}{ll}p & 0 \\
q & 0\end{array}\right)$ \\
\hline $\begin{array}{l}\mathrm{I}_{\mathbb{C}} \\
\mathrm{I}_{\mathbb{C}}^{c}\end{array}$ & $\begin{array}{l}\mathfrak{u}(p+1, q+1) \\
\mathfrak{u}(p+q+1,1)\end{array}$ & $\mathfrak{u}(q)+\mathfrak{u}(p+1,1)$ & $\left(\begin{array}{cc}2 p & 1 \\
2 q & 0\end{array}\right)$ \\
\hline $\begin{array}{l}\mathrm{I}_{\mathbb{H}} \\
\mathrm{I}_{\mathbb{H}}^{c}\end{array}$ & $\begin{array}{l}\mathfrak{s p}(p+1, q+1) \\
\mathfrak{s p}(p+q+1,1) \\
\end{array}$ & $\mathfrak{s p}(q)+\mathfrak{s p}(p+1,1)$ & $\left(\begin{array}{cc}4 p & 3 \\
4 q & 0\end{array}\right)$ \\
\hline \multirow[t]{4}{*}{$\mathrm{I}_{\mathbb{O}}=\mathrm{I}_{\mathbb{O}}^{c}$} & $\mathfrak{f}_{4(-20)}$ & $\mathfrak{s o}(8,1)$ & $\left(\begin{array}{ll}0 & 7 \\
8 & 0\end{array}\right)$ \\
\hline & $\begin{array}{l}\mathfrak{s l}(m+2, \mathbb{R}) \\
\mathfrak{s l}(m+1, \mathbb{R})\end{array}$ & $\mathfrak{s o}(m+1,1)$ & $\left(\begin{array}{ll}m & 0 \\
m & 1\end{array}\right)$ \\
\hline & $\begin{array}{l}\mathfrak{s p}(m+2, \mathbb{R}) \\
\mathfrak{s p}(m+1, \mathbb{R})\end{array}$ & $\mathfrak{u}(m+1,1)$ & $\left(\begin{array}{ll}2 m & 1 \\
2 m & 2\end{array}\right)$ \\
\hline & $\begin{array}{c}\mathfrak{f}_{4(4)} \\
\mathfrak{f}_{4(-20)}\end{array}$ & $\mathfrak{s p}(2,1)+\mathfrak{s u}(2)$ & $\left(\begin{array}{ll}4 & 3 \\
4 & 4\end{array}\right)$ \\
\hline \multirow[t]{4}{*}{$\begin{array}{c}\text { II } \\
\text { II }^{c}\end{array}$} & $\begin{array}{c}\mathfrak{s o}(m+2, \mathbb{C}) \\
\mathfrak{h}+\mathfrak{h}\end{array}$ & $\mathfrak{s o}(m+1,1)$ & $\left(\begin{array}{ll}m & 0 \\
m & 0\end{array}\right)$ \\
\hline & $\begin{array}{c}\mathfrak{s l}(m+2, \mathbb{C}) \\
\mathfrak{h}+\mathfrak{h}\end{array}$ & $\mathfrak{s u}(m+1,1)$ & $\left.\begin{array}{ll}2 m & 1 \\
2 m & 1\end{array}\right)$ \\
\hline & $\begin{array}{c}\mathfrak{s p}(m+2, \mathbb{C}) \\
\mathfrak{h}+\mathfrak{h}\end{array}$ & $\mathfrak{s p}(m+1,1)$ & $\left(\begin{array}{ll}4 m & 3 \\
4 m & 3\end{array}\right)$ \\
\hline & $\begin{array}{l}\mathfrak{f}_{4}(\mathbb{C}) \\
\mathfrak{h}+\mathfrak{h}\end{array}$ & $\mathfrak{f}_{4(-20)}$ & $\left(\begin{array}{ll}8 & 7 \\
8 & 7\end{array}\right)$ \\
\hline \multirow[t]{6}{*}{$\begin{array}{c}\text { III } \\
\text { III }^{c} \\
\end{array}$} & $\begin{array}{c}\mathfrak{s o}^{*}(2 m+4) \\
\mathfrak{s o}(2 m+2,2)\end{array}$ & $\mathfrak{u}(m+1,1)$ & $\left.\begin{array}{ll}2 m & 1 \\
2 m & 0\end{array}\right)$ \\
\hline & $\begin{array}{c}\mathfrak{s u}^{*}(2 m+4) \\
\mathfrak{s u}(2 m+2,2)\end{array}$ & $\mathfrak{s p}(m+1,1)$ & $\left.\begin{array}{ll}4 m & 3 \\
4 m & 1\end{array}\right)$ \\
\hline & $\begin{array}{l}\mathfrak{e}_{6(-26)} \\
\mathfrak{e}_{6(-14)}\end{array}$ & $\mathfrak{f}_{4(-20)}$ & $\left(\begin{array}{ll}8 & 7 \\
8 & 1\end{array}\right)$ \\
\hline & $\mathfrak{s l}(3, \mathbb{C})$ & $\mathfrak{s o}(3, \mathbb{C})$ & $\left(\begin{array}{ll}2 & 0 \\
2 & 2\end{array}\right)$ \\
\hline & $\begin{array}{c}\mathfrak{s u}(3,3) \\
\mathfrak{s u}^{*}(6) \\
\end{array}$ & $\mathfrak{s o}^{*}(6)$ & $\left(\begin{array}{ll}4 & 1 \\
4 & 3\end{array}\right)$ \\
\hline & $\begin{array}{c}\mathfrak{e}_{6(2)} \\
\mathfrak{e}_{6(-26)}\end{array}$ & $\mathfrak{s} \not(3,1)$ & $\left(\begin{array}{ll}8 & 3 \\
8 & 5\end{array}\right)$ \\
\hline
\end{tabular}


Table 6.2: Irreducible symmetric pairs with (QP) and $\operatorname{rank}_{\mathbb{R}} \mathfrak{h}=1$

\begin{tabular}{|c|c|c|c|}
\hline & $\left(\mathfrak{m}_{H} \cap \mathfrak{m}_{G}\right)+\mathfrak{a}_{H}$ & $\mathfrak{m}_{H}+\mathfrak{a}_{H}$ & $\mathfrak{n}^{-\sigma}$ \\
\hline $\mathrm{I}_{\mathbb{R}}$ & $\mathfrak{o}(|p-q|)+\mathbb{R}$ & \multirow{2}{*}{$\mathfrak{o}(q)+\mathfrak{o}(p)+\mathbb{R}$} & \multirow{2}{*}{$\mathbb{R}^{q}$} \\
\hline $\mathrm{I}_{\mathbb{R}}^{c}$ & $\mathfrak{o}(q)+\mathfrak{o}(p)+\mathbb{R}$ & & \\
\hline $\mathrm{I}_{\mathbb{C}}$ & $\mathfrak{u}(|p-q|)+(\sqrt{-1} \mathbb{R})^{\min (p, q)}+\mathbb{R}$ & \multirow{2}{*}{$\mathfrak{u}(q)+\mathfrak{u}(p)+\mathbb{C}$} & \multirow{2}{*}{$\mathbb{C}^{q}$} \\
\hline $\mathrm{I}_{\mathbb{C}}^{c}$ & $\mathfrak{u}(q)+\mathfrak{u}(p)+\mathbb{C}$ & & \\
\hline $\mathrm{I}_{\mathbb{H}}$ & $\mathfrak{s p}(|p-q|)+\mathfrak{s p}(1)^{\min (p, q)}+\mathbb{R}$ & \multirow{2}{*}{$\mathfrak{s p}(q)+\mathfrak{s p}(p)+\mathbb{H}$} & \multirow{2}{*}{$\mathbb{H}^{q}$} \\
\hline $\mathrm{I}_{\mathbb{H}}^{c}$ & $\mathfrak{s p}(q)+\mathfrak{s p}(p)+\mathbb{H}$ & & \\
\hline $\mathrm{I}_{\mathbb{O}}=\mathrm{I}_{\mathbb{O}}^{c}$ & $\mathfrak{s p i n}(7)+\mathbb{R}$ & $\mathfrak{s p i n}(7)+\mathbb{R}$ & $\mathbb{R}^{8}$ \\
\hline II & $\mathbb{T}^{\left[\frac{m}{2}\right]}+\mathbb{R}$ & \multirow{2}{*}{$\mathfrak{o}(m)+\mathbb{R}$} & \multirow{2}{*}{$\mathbb{R}^{m}$} \\
\hline $\mathrm{II}^{c}$ & $\mathfrak{o}(m)+\mathbb{R}$ & & \\
\hline III & $\mathfrak{s p}(1)^{\left[\frac{m}{2}\right]}+\mathbb{C}$ & \multirow{2}{*}{$\mathfrak{u}(m)+\mathbb{C}$} & \multirow{2}{*}{$\mathbb{C}^{m}$} \\
\hline $\mathrm{III}^{c}$ & $\mathfrak{u}(m)+\mathbb{R}$ & & \\
\hline
\end{tabular}

\section{Associated symmetric pairs of non- $K_{\varepsilon}$-family}

In this section and the next section, we complete the proof of the classification of symmetric pairs $(\mathfrak{g}, \mathfrak{h})$ satisfying $(\mathrm{PP})$ (or $(\mathrm{QP})$ ) and $\operatorname{rank}_{\mathbb{R}} H \geq 2$. For this, we make use of the $K_{\varepsilon}$-family introduced in [18] (See Definition 7.1 below), which is a fairly large class of reductive symmetric pairs.

We recall that if a reductive symmetric pair $(\mathfrak{g}, \mathfrak{h})$ is defined by an involutive automorphism $\sigma$ of $\mathfrak{g}$ then we can define another involution $\sigma \theta$ by taking a Cartan involution $\theta$ commuting with $\sigma$. The symmetric pair $\left(\mathfrak{g}, \mathfrak{g}^{\sigma \theta}\right)$ is called the associated symmetric pair of $(\mathfrak{g}, \mathfrak{h}) \equiv\left(\mathfrak{g}, \mathfrak{g}^{\sigma}\right)$. Our strategy is based on the following ideas.

(1) Very few pairs $(\mathfrak{g}, \mathfrak{h}) \equiv\left(\mathfrak{g}, \mathfrak{g}^{\sigma}\right)$ satisfy $(\mathrm{QP})$ if $\left(\mathfrak{g}, \mathfrak{g}^{\sigma \theta}\right)$ does not belong to the $K_{\varepsilon}$-family (Proposition 7.2).

(2) $\operatorname{rank}_{\mathbb{R}} G=\operatorname{rank}_{\mathbb{R}} H$ if $\left(\mathfrak{g}, \mathfrak{g}^{\sigma \theta}\right)$ belongs to the $K_{\varepsilon}$-family.

In this section we treat the case where the associated symmetric pair $\left(\mathfrak{g}, \mathfrak{g}^{\sigma \theta}\right)$ does not belong to the $K_{\varepsilon}$-family, and in the next section we discuss the opposite case where $\left(\mathfrak{g}, \mathfrak{g}^{\sigma \theta}\right)$ belongs to the $K_{\varepsilon}$-family. To be more precise, let us review the definition of $K_{\varepsilon}$-family. Suppose $\mathfrak{a}_{G}$ is a maximal abelian subspace of $\mathfrak{g}^{-\theta}$ as before.

Definition 7.1. A map $\varepsilon: \Sigma\left(\mathfrak{g}, \mathfrak{a}_{G}\right) \cup\{0\} \rightarrow\{ \pm 1\}$ is said to be a signature if

$$
\varepsilon(\alpha+\beta)=\varepsilon(\alpha) \varepsilon(\beta) \quad \text { for any } \alpha, \beta \text { and } \alpha+\beta \in \Sigma\left(\mathfrak{g}, \mathfrak{a}_{G}\right) \cup\{0\} \text {. }
$$


We note that $\varepsilon(0)=1$ and $\varepsilon(\alpha)=\varepsilon(-\alpha)$ for any $\alpha \in \Sigma\left(\mathfrak{g}, \mathfrak{a}_{G}\right)$. We define another involution $\theta_{\varepsilon}$ by

$$
\theta_{\varepsilon}(X):=\varepsilon(\alpha) \theta(X) \quad \text { for } \quad X \in \mathfrak{g}\left(\mathfrak{a}_{G} ; \alpha\right),
$$

and set $\mathfrak{k}_{\varepsilon}:=\left\{X \in \mathfrak{g}: \theta_{\varepsilon}(X)=X\right\}$. If $\varepsilon \equiv 1$ then $\mathfrak{k}_{\varepsilon}=\mathfrak{k}$. The reductive symmetric pairs $\left\{\left(\mathfrak{g}, \mathfrak{k}_{\varepsilon}\right): \varepsilon\right.$ is a signature $\}$ are called the $K_{\varepsilon}$-family.

Here is the main result of this section:

Proposition 7.2. Let $(G, H)$ be an irreducible symmetric pair defined by an involution $\sigma$. Assume that the following two conditions are fulfilled:

$$
\begin{aligned}
& \text { The associated pair }\left(\mathfrak{g}, \mathfrak{g}^{\sigma \theta}\right) \text { does not belong to the } K_{\varepsilon} \text {-family. } \\
& \operatorname{rank}_{\mathbb{R}} H>1 \text {. }
\end{aligned}
$$

Then either $\operatorname{rank}_{\mathbb{R}} H<\# \Delta\left(\mathfrak{n}^{-\sigma}\right)$ or

$(\mathfrak{g}, \mathfrak{h})$ is a symmetric pair treated in Propositions 5.1, 5.8 and 5.7.

In particular, there is no irreducible symmetric pair $(\mathfrak{g}, \mathfrak{h})$ with (7.1) and (7.2) other than those listed in Propositions 5.1, 5.0 and 5.7.

Proof. Suppose $\left(\mathfrak{g}, \mathfrak{g}^{\sigma \theta}\right)$ does not belong to the $K_{\varepsilon}$-family. Then by using the classification [19, Table V] and by computing the correspondence $(\mathfrak{g}, \mathfrak{h}) \equiv\left(\mathfrak{g}, \mathfrak{g}^{\sigma}\right) \leftrightarrow\left(\mathfrak{g}, \mathfrak{g}^{\sigma \theta}\right)$, we observe that either $H$ is a simple Lie group up to a compact torus or (7.3) holds.

From now on, we assume that the irreducible symmetric pair $(\mathfrak{g}, \mathfrak{h})$ satisfies $(7.1)$ and (7.2) but does not satisfy (7.3). Then the restricted root system $\Sigma\left(\mathfrak{h}, \mathfrak{a}_{H}\right)$ is irreducible. Then, by Lemma 3.13, the condition $\operatorname{rank}_{\mathbb{R}} H \geq \# \Delta\left(\mathfrak{n}^{-\sigma}\right)$ gives strong constraints on both the irreducible root system $\Sigma\left(\mathfrak{h}, \mathfrak{a}_{H}\right)$ and the set $\Delta\left(\mathfrak{n}^{-\sigma}\right)$, namely, $\operatorname{rank}_{\mathbb{R}} H \geq \# \Delta\left(\mathfrak{n}^{-\sigma}\right)$ implies that $\Sigma\left(\mathfrak{h}, \mathfrak{a}_{H}\right)$ is one of type $B_{l}, C_{l}, D_{l}$ or $B C_{l}$ and that $\Delta\left(\mathfrak{n}^{-\sigma}\right)$ is contained in either $\left\{ \pm e_{i}: 1 \leq i \leq l\right\}$ or $\left\{ \pm 2 e_{i}: 1 \leq i \leq l\right\}$. Furthermore, $m^{-}(\lambda) \leq 1$ and $m^{-}(\lambda) m^{-}(2 \lambda)=0$ for all $\lambda$.

In turn, in view of the classification of irreducible symmetric pairs satisfying (7.1) and the formulae for the multiplicities $m^{-}\left(\lambda_{i}\right)$ and $m^{-}\left(2 \lambda_{i}\right)$ in [19, Table V], we see that this does not happen. To verify it, we remark that the role of $\left(\mathfrak{g}, \mathfrak{g}^{\sigma}\right)$ and $\left(\mathfrak{g}, \mathfrak{g}^{\sigma \theta}\right)$ in their table is opposite to our notation here, but the role of the multiplicities $m^{ \pm}(\lambda)$ is the same. With this remark in mind, we obtain the following small list from 19 , Table V] by picking up those having the above constraints on $\Sigma\left(\mathfrak{h}, \mathfrak{a}_{H}\right)$ and $\Delta\left(\mathfrak{n}^{-\sigma}\right)$ 
and by skipping those belonging to the families in Proposition 5.1, 5.6 and 5.7:

$\begin{array}{lll}\mathfrak{g} & \mathfrak{g}^{\sigma \theta} & \mathfrak{g}^{\sigma}=\mathfrak{h} \\ \mathfrak{s l}(4, \mathbb{R}) & \mathfrak{s l}(2, \mathbb{C})+\sqrt{-1} \mathbb{R} & \mathfrak{s p}(2, \mathbb{R}) \\ \mathfrak{s u}(2,2) & \mathfrak{s o}^{*}(4) & \mathfrak{s p}(2, \mathbb{R}) \\ \mathfrak{s o}^{*}(8) & \mathfrak{s o}^{*}(4)+\mathfrak{s o}^{*}(4) & \mathfrak{u}(2,2) \\ \mathfrak{s o}(4,4) & \mathfrak{u}(2,2) & \mathfrak{u}(2,2) \\ \mathfrak{s l}(4, \mathbb{C}) & \mathfrak{s u}(4) & \mathfrak{s p}(2, \mathbb{C})\end{array}$

However, these exceptional cases are actually included in the family of symmetric pairs in Propositions 5.1 and 5.6 via the following isomorphisms:

$$
\begin{aligned}
(\mathfrak{s l}(4, \mathbb{R}), \mathfrak{s p}(2, \mathbb{R})) & \simeq(\mathfrak{s o}(3,3), \mathfrak{s o}(3,2)), \\
(\mathfrak{s u}(2,2), \mathfrak{s p}(2, \mathbb{R})) & \simeq(\mathfrak{s o}(4,2), \mathfrak{s o}(3,2)), \\
\left(\mathfrak{s o} *^{*}(8), \mathfrak{u}(2,2)\right) & \simeq(\mathfrak{s o}(6,2), \mathfrak{s o}(4,2)+\mathfrak{s o}(2)), \\
(\mathfrak{s o}(4,4), \mathfrak{u}(2,2)) & \simeq(\mathfrak{s o}(4,4), \mathfrak{s o}(4,2)+\mathfrak{s o}(2)), \\
(\mathfrak{s l}(4, \mathbb{C}), \mathfrak{s p}(2, \mathbb{C})) & \simeq(\mathfrak{s o}(6, \mathbb{C}), \mathfrak{s o}(5, \mathbb{C})) .
\end{aligned}
$$

Thus we have proved that $\operatorname{rank}_{\mathbb{R}} H<\# \Delta\left(\mathfrak{n}^{-\sigma}\right)$ if $(\overline{7.1})$, and $(\overline{7.2})$ are satisfied and if (7.3) is not satisfied.

\section{Associated symmetric pairs of $K_{\varepsilon}$-family}

In this section we consider irreducible symmetric pairs $(\mathfrak{g}, \mathfrak{h}) \equiv\left(\mathfrak{g}, \mathfrak{g}^{\sigma}\right)$ such that the associated symmetric pair $\left(\mathfrak{g}, \mathfrak{g}^{\sigma \theta}\right)$ belongs to the $K_{\varepsilon}$-family. In this case, $\operatorname{rank}_{\mathbb{R}} H=$ $\operatorname{rank}_{\mathbb{R}} G$ holds from the definition of $K_{\varepsilon}$-family, and consequently, the condition (QP) is equivalent to $(\mathrm{PP})$ by Lemma 3.1 .

Let $\mathfrak{g}_{\mathbb{C}}$ be the complexification of $\mathfrak{g}$. For a simple Lie algebra $\mathfrak{g}$ over $\mathbb{R}, \mathfrak{g}_{\mathbb{C}}$ is a complex simple Lie algebra if and only if $\mathfrak{g}$ itself does not carry a complex Lie algebra structure. We divide the proof into the following three cases:

Case 1. $\mathfrak{g}_{\mathbb{C}}$ is not simple.

Case 2. $\mathfrak{g}_{\mathbb{C}}$ is a simple classical Lie algebra.

Case 3. $\mathfrak{g}_{\mathbb{C}}$ is a simple exceptional Lie algebra.

In Case 1 , the pair $(\mathfrak{g}, \mathfrak{h})$ was treated in Proposition 4.3. In fact, $\mathfrak{g}$ is a complex simple Lie algebra. Further, $\mathfrak{g}^{\sigma \theta}$ is a real form of $\mathfrak{g}$ as noted in [18, Appendix], and consequently $\mathfrak{h}=\mathfrak{g}^{\sigma}$ is a complex Lie subalgebra. Hence $(\mathfrak{g}, \mathfrak{h})$ is a complex symmetric pair such that rank $\mathfrak{h}=\operatorname{rank} \mathfrak{g}$. 
Case 2. Suppose that $\mathfrak{g}_{\mathbb{C}}$ is a classical simple Lie algebra.

By the classification of $K_{\varepsilon}$-family (see [19, Table 1]), the pair $(\mathfrak{g}, \mathfrak{h})$ is one of the following pairs up to the center of $\mathfrak{g}$.

$$
\begin{aligned}
& (\mathfrak{g l}(p+q, \mathbb{F}), \mathfrak{g l}(p, \mathbb{F})+\mathfrak{g l}(q, \mathbb{F})), \quad \mathbb{F}=\mathbb{R}, \mathbb{H}, \\
& (\mathfrak{s p}(p+q, \mathbb{R}), \mathfrak{s p}(p, \mathbb{R})+\mathfrak{s p}(q, \mathbb{R})), \\
& (\mathfrak{u}(n, n ; \mathbb{F}), \mathfrak{g l}(n, \mathbb{F})), \quad \mathbb{F}=\mathbb{R}, \mathbb{C}, \mathbb{H}, \\
& (\mathfrak{s p}(n, \mathbb{R}), \mathfrak{g l}(n, \mathbb{R})), \\
& \left(\mathfrak{s o}^{*}(4 n), \mathfrak{g l}(n, \mathbb{H})\right),
\end{aligned}
$$

or the following two families

$$
\begin{aligned}
& (\mathfrak{u}(i+j, k+l ; \mathbb{F}), \mathfrak{u}(i, k ; \mathbb{F})+\mathfrak{u}(j, l ; \mathbb{F})), \quad \mathbb{F}=\mathbb{R}, \mathbb{C}, \mathbb{H}, \\
& \left(\mathfrak{o}^{*}(2 p+2 q), \mathfrak{o}^{*}(2 p)+\mathfrak{o}^{*}(2 q)\right) .
\end{aligned}
$$

In the last two cases, the condition that $\left(\mathfrak{g}, \mathfrak{g}^{\sigma \theta}\right)$ belongs to the $K_{\varepsilon}$-family imposes certain constraints on the parameters (e.g. $p q$ is even in the last case).

The first five cases were treated in Propositions 5.4, 5.12, 5.9, and 5.11. The last two cases are covered by Propositions 5.1 and 5.7 without constraints on parameters, respectively. Thus there is no "new" symmetric pair $(\mathfrak{g}, \mathfrak{h})$ that satisfies (QP).

Case 3. $\mathfrak{g}_{\mathbb{C}}$ is an exceptional simple Lie algebra.

In this case, we prove the following:

Proposition 8.1. Let $(\mathfrak{g}, \mathfrak{h}) \equiv\left(\mathfrak{g}, \mathfrak{g}^{\sigma}\right)$ be a symmetric pair such that its associated symmetric pair $\left(\mathfrak{g}, \mathfrak{g}^{\sigma \theta}\right)$ belongs to the $K_{\varepsilon}$-family. If $\mathfrak{g}_{\mathbb{C}}$ is a simple exceptional Lie algebra, then the following three conditions are equivalent:

(i) $(\mathfrak{g}, \mathfrak{h})$ satisfies $(\mathrm{QP})$.

(ii) $(\mathfrak{g}, \mathfrak{h})$ satisfies $(\mathrm{PP})$.

(iii) $(\mathfrak{g}, \mathfrak{h})$ is either $\left(\mathfrak{e}_{6(-26)}, \mathfrak{s o}(9,1)+\mathbb{R}\right)$ or $\left(\mathfrak{f}_{4(-20)}, \mathfrak{s o}(8,1)\right)$.

Proof. The equivalence (i) $\Leftrightarrow$ (ii) holds because $\operatorname{rank}_{\mathbb{R}} G=\operatorname{rank}_{\mathbb{R}} H$. We have already proved the implication (iii) $\Rightarrow$ (ii) in Propositions 5.14 and 6.2. The remaining implication (i) $\Rightarrow$ (iii) is deduced from the following two lemmas.

Lemma 8.2. The symmetric pair $\left(\mathfrak{e}_{6(-14)}, \mathfrak{s o}(8,2)+\sqrt{-1} \mathbb{R}\right)$ does not satisfy $(\mathrm{QP})$. 
Proof. We take the standard basis $\left\{e_{1}, e_{2}\right\}$ of $\mathfrak{a}_{H}^{*}=\mathfrak{a}_{G}^{*}$ in such a way that $\Sigma^{+}\left(\mathfrak{h}, \mathfrak{a}_{H}\right)=$ $\left\{e_{1}, e_{2}, e_{1} \pm e_{2}\right\}$. Then the root multiplicities $m^{ \pm}(\lambda)$ are given as follows:

$\begin{array}{lccc}\lambda & \pm e_{i}(i=1,2) & \pm 2 e_{i}(i=1,2) & \pm e_{1} \pm e_{2} \\ m^{+}(\lambda) & 6 & 0 & 1 \\ m^{-}(\lambda) & 0 & 1 & 7\end{array}$

Thus $\Delta\left(\mathfrak{n}^{-\sigma}\right)=\left\{2 e_{1}, 2 e_{2}, e_{1} \pm e_{2}\right\}$, and $\# \Delta\left(\mathfrak{n}^{-\sigma}\right)=4>\operatorname{rank}_{\mathbb{R}} H=2$. Now the lemma follows from Proposition 3.11.

For the remaining cases, we use Proposition 3.14 as an easy-to-check sufficient condition for (QP). We obtain the following:

Lemma 8.3. Let $\mathfrak{g}$ be an exceptional simple Lie algebra and $(\mathfrak{g}, \mathfrak{h})$ a symmetric pair such that its associated symmetric pair belongs to the $K_{\varepsilon}$-family. Then the inequality (3.9) holds if and only if the pair $(\mathfrak{g}, \mathfrak{h})$ is one of the following:

$$
\left(\mathfrak{e}_{6(-14)}, \mathfrak{s o}(8,2)+\sqrt{-1} \mathbb{R}\right), \quad\left(\mathfrak{e}_{6(-26)}, \mathfrak{s o}(9,1)+\mathbb{R}\right), \quad\left(\mathfrak{f}_{4(-20)}, \mathfrak{s o}(8,1)\right)
$$

Proof. In Table 8.1, we list all the symmetric pairs $(\mathfrak{g}, \mathfrak{h}) \equiv\left(\mathfrak{g}, \mathfrak{g}^{\sigma}\right)$ such that $\mathfrak{g}_{\mathbb{C}}$ is an exceptional simple Lie algebra and that $\left(\mathfrak{g}, \mathfrak{g}^{\sigma \theta}\right)$ belongs to the $K_{\varepsilon}$-family. In this table, we also list the data $m(G)$ (see (3.7)); $n(G), n(H)$ (see (3.8)), and $\operatorname{rank}_{\mathbb{R}} G\left(=\operatorname{rank}_{\mathbb{R}} H\right.$ ). Now Lemma 8.3 follows from the computation of the signature of $n(G)-n(H)-$ $m(G) \operatorname{rank}_{\mathbb{R}} H$. 
Table 8.1: Exceptional symmetric pairs $(\mathfrak{g}, \mathfrak{h}) \equiv\left(\mathfrak{g}, \mathfrak{g}^{\sigma}\right)$ with $\left(\mathfrak{g}, \mathfrak{g}^{\sigma \theta}\right)$ in $K_{\varepsilon}$-family

\begin{tabular}{|c|c|c|c|c|c|c|}
\hline$G$ & $\operatorname{rank}_{\mathbb{R}} G$ & $m(G)$ & $n(G)$ & $H$ & $n(H)$ & $m(G) \operatorname{rank}_{\mathbb{R}} G$ v.s. $n(G)-n(H)$ \\
\hline \multirow[b]{2}{*}{$\mathfrak{e}_{6(6)}$} & \multirow{2}{*}{6} & \multirow{2}{*}{1} & \multirow{2}{*}{36} & $\mathfrak{s l}(6, \mathbb{R})+\mathfrak{s l}(2, \mathbb{R})$ & 16 & $6<20$ \\
\hline & & & & $\mathfrak{s o}(5,5)+\mathbb{R}$ & 20 & $6<16$ \\
\hline \multirow[b]{2}{*}{$\mathfrak{e}_{6(2)}$} & \multirow[b]{2}{*}{4} & \multirow[b]{2}{*}{2} & \multirow[b]{2}{*}{36} & $\mathfrak{s o}(6,4)+\sqrt{-1} \mathbb{R}$ & 20 & $8<16$ \\
\hline & & & & $\mathfrak{s u}(3,3)+\mathfrak{s l}(2, \mathbb{R})$ & 16 & $8<20$ \\
\hline \multirow[b]{2}{*}{$\mathfrak{e}_{6}(-14)$} & \multirow{2}{*}{2} & \multirow{2}{*}{8} & \multirow{2}{*}{30} & $\mathfrak{s u}(5,1)+\mathfrak{s l}(2, \mathbb{R})$ & 10 & $16<20$ \\
\hline & & & & $\mathfrak{s o}(8,2)+\sqrt{-1} \mathbb{R}$ & 14 & $16=16$ \\
\hline $\mathfrak{e}_{6(-26)}$ & 2 & 8 & 24 & $\mathfrak{s o}(9,1)+\mathbb{R}$ & 8 & $16>8$ \\
\hline \multirow{3}{*}{$\mathfrak{e}_{7(7)}$} & \multirow{3}{*}{7} & \multirow{3}{*}{1} & \multirow{3}{*}{63} & $\mathfrak{s l}(8, \mathbb{R})$ & 28 & $7<35$ \\
\hline & & & & $\mathfrak{s o}(6,6)+\mathfrak{s l}(2, \mathbb{R})$ & 31 & $7<32$ \\
\hline & & & & $\mathfrak{e}_{6(6)}+\mathbb{R}$ & 36 & $7<27$ \\
\hline \multirow[b]{2}{*}{$\mathfrak{e}_{7(-5)}$} & \multirow{2}{*}{4} & \multirow{2}{*}{4} & \multirow{2}{*}{60} & $\mathfrak{s o}(8,4)+\mathfrak{s u}(2)$ & 28 & $16<32$ \\
\hline & & & & $\mathfrak{s o}^{*}(12)+\mathfrak{s l}(2, \mathbb{R})$ & 28 & $16<32$ \\
\hline \multirow{2}{*}{$\mathfrak{e}_{7(-25)}$} & \multirow{2}{*}{3} & \multirow{2}{*}{8} & \multirow{2}{*}{51} & $\overline{\mathfrak{e}_{6(-26)}+\mathbb{R}}$ & 24 & $24<27$ \\
\hline & & & & $\mathfrak{s o}(10,2)+\mathfrak{s l}(2, \mathbb{R})$ & 19 & $24<32$ \\
\hline \multirow{2}{*}{$\mathfrak{e}_{8(8)}$} & \multirow{2}{*}{8} & \multirow{2}{*}{1} & \multirow{2}{*}{120} & $\mathfrak{s o}(8,8)$ & 56 & $8<64$ \\
\hline & & & & $\mathfrak{e}_{7(7)}+\mathfrak{s l}(2, \mathbb{R})$ & 64 & $8<56$ \\
\hline \multirow[b]{2}{*}{$\mathfrak{e}_{8(-24)}$} & \multirow{2}{*}{4} & \multirow{2}{*}{8} & \multirow{2}{*}{108} & $\mathfrak{s o}(12,4)$ & 44 & $32<64$ \\
\hline & & & & $\mathfrak{e}_{7(-25)}+\mathfrak{s l}(2, \mathbb{R})$ & 52 & $32<56$ \\
\hline \multirow{2}{*}{$\mathfrak{f}_{4(4)}$} & \multirow{2}{*}{4} & & & $\mathfrak{s o}(5,4)$ & 16 & $4<8$ \\
\hline & & 1 & 24 & $\mathfrak{s p}(3, \mathbb{R})+\mathfrak{s l}(2, \mathbb{R})$ & 10 & $4<14$ \\
\hline $\mathfrak{f}_{4(-20)}$ & 1 & 8 & 15 & $\mathfrak{s o}(8,1)$ & 7 & $8=8$ \\
\hline $\mathfrak{g}_{2(2)}$ & 2 & 1 & 6 & $\mathfrak{s l}(2, \mathbb{R})+\mathfrak{s l}(2, \mathbb{R})$ & 2 & $2<4$ \\
\hline
\end{tabular}

\section{Applications to branching problems}

This section is devoted to applications of our classification results (Theorem 1.3 and Proposition 1.6) to branching problems of real reductive groups. Given an irreducible representation $\pi$ of $G$, we wish to understand how the representation $\pi$ behaves as a representation of a subgroup $H$ (branching problems). Basic quantities are the dimension of continuous $H$-homomorphisms

$$
m(\pi, \tau):=\operatorname{dim} \operatorname{Hom}_{H}\left(\left.\pi\right|_{H}, \tau\right),
$$

for irreducible representations $\tau$ of $H$. Concrete analysis of the restriction $\left.\pi\right|_{H}$ could be reasonably developed under the condition that $m(\pi, \tau)<\infty$. However, the finiteness 
of the multiplicities does not always hold even when $H$ is a maximal subgroup of $G$ (see [9, 11] for good behaviors and bad behaviors of the restriction $\left.\pi\right|_{H}$ ). The initial motivation of our work is to single out a nice framework on the pair $(G, H)$ of reductive groups for which we could expect that the branching laws $\left.\pi\right|_{H}$ behave reasonably for arbitrary irreducible representations $\pi$.

\subsection{Admissible smooth representations}

We begin with a quick review of some basic notion of (infinite-dimensional) continuous representations of real reductive groups.

Suppose $G$ is a real reductive linear Lie group (or its finite cover) and $K$ is a maximal compact subgroup.

Let $\pi$ be a continuous representation of $G$ on a complete, locally convex vector space $\mathcal{H}$. The space $\mathcal{H}^{\infty}$ of $C^{\infty}$-vectors of $(\pi, \mathcal{H})$ is naturally endowed with Fréchet topology, and we obtain a continuous representation $\pi^{\infty}$ of $G$ on $\mathcal{H}^{\infty}$.

Suppose that $(\pi, \mathcal{H})$ is of finite length, in other words, suppose that there are only finitely many closed invariant subspaces in $\mathcal{H}$. We say $\pi$ is admissible (or $K$-admissible) if

$$
\operatorname{dim} \operatorname{Hom}_{K}\left(\tau,\left.\pi\right|_{K}\right)<\infty
$$

for any irreducible finite-dimensional representation $\tau$ of $K$. For an admissible representation $(\pi, \mathcal{H})$ such that $\mathcal{H}$ is a Banach space, we say $\left(\pi^{\infty}, \mathcal{H}^{\infty}\right)$ is an admissible smooth representation. By the Casselman-Wallach globalization theory, there is a canonical equivalence of categories between the category of $(\mathfrak{g}, K)$-modules of finite length and the category of admissible smooth representations of $G$. An admissible smooth representation is sometimes referred to as a smooth Fréchet representation of moderate growth ([21, Chapter 11]). An irreducible admissible smooth representation of $G$ is said to be an irreducible smooth representation for simplicity throughout this article.

\subsection{Finite multiplicity property in branching laws}

Suppose that $G$ is a real reductive linear Lie group and $H$ is a reductive subgroup defined algebraically over $\mathbb{R}$. In what follows, the results remain true if we replace $(G, H)$ by their finite coverings or by their finite-index subgroups. Following the terminology in [13, we formulate a finite-multiplicity property on the pair $(G, H)$ for the restriction of admissible representations:

$(\mathbf{F M})$ (Finite-multiplicity property) $\operatorname{dim} \operatorname{Hom}_{H}\left(\left.\pi\right|_{H}, \tau\right)<\infty$, for any admissible smooth representation $\pi$ of $G$ and for any admissible smooth representation $\tau$ of $H$. 
Here $\operatorname{Hom}_{H}($,$) denotes the space of continuous H$-homomorphisms.

As a direct consequence of Theorem 1.3 and Fact 1.8, we obtain a complete classification of the reductive symmetric pairs $(G, H)$ having the finite-multiplicity property $(\mathrm{FM})$.

Theorem 9.1. Suppose $(G, H)$ is a reductive symmetric pair. Then the following two conditions are equivalent:

(i) $(G, H)$ satisfies the finite-multiplicity property $(\mathrm{FM})$ for the restriction of admissible smooth representations.

(ii) The pair $(\mathfrak{g}, \mathfrak{h})$ of Lie algebras is isomorphic to the direct sum of the pairs $(\mathrm{A})-(\mathrm{H})$ in Theorem 1.3 up to outer automorphisms.

Remark 9.2. Here are some features of the implication (ii) $\Rightarrow$ (i) in Theorem 9.1 for the following special settings among $(\mathrm{A})-(\mathrm{H})$ :

1) For the pairs $(\mathrm{B})$ and $(\mathrm{C})$, the finite-multiplicity property $(\mathrm{FM})$ is obvious because $\pi$ is a finite-dimensional representation.

2) For the pairs (D) (i.e. $H=K$ ), the finite-multiplicity property (FM) is trivial by the definition of admissible representations. (However, there are a number of equivalent conditions on admissibility, and the proof of Fact 1.8 given in 13. is not a tautology for $H=K$ but includes a microlocal proof of the classical fact that quasisimple irreducible representations are admissible, which was first proved by Harish-Chandra [7].)

3) For the pairs (F), we have a uniform estimate of the multiplicities, as we shall see in Subsection 9.3.

4) For the pairs $(\mathrm{G})$, i.e., $(G, H)=\left(G^{\prime} \times G^{\prime}, \operatorname{diag} G^{\prime}\right)$, the finite-multiplicity property $(\mathrm{FM})$ can be interpreted as the finiteness of linearly independent invariant trilinear forms, see Subsection 9.4.

Remark 9.3. The property (FM) is a condition on the pair $(G, H)$ of groups that assures the finiteness of the multiplicity $m(\pi, \tau)$ for arbitrary $\pi$ and $\tau$. On the other hand, we may discuss a condition on the triple $(G, H, \pi)$ for which $m(\pi, \tau)$ is finite for arbitrary $\tau$. This direction was pursued in [9] under the additional assumption of discrete decomposability of branching laws (referred to as $H$-admissible restriction), and the classification theory has been recently studied in [14, 15, particularly for "relatively small" infinite-dimensional representations $\pi$ of $G$ (e.g., Zuckerman's derived functor modules, minimal representations, etc.). 


\subsection{Uniformly bounded multiplicities}

In addition to the aforementioned finite-multiplicity property (FM), we consider the following two properties on a pair of reductive groups $(G, H)$ :

(BM) (Bounded-multiplicity restriction) There exists a constant $C<\infty$ such that

$$
\operatorname{dim} \operatorname{Hom}_{H}\left(\left.\pi\right|_{H}, \tau\right) \leq C
$$

for any irreducible smooth representation $\pi$ of $G$ and for any irreducible smooth representation $\tau$ of $H$.

(MF) (Multiplicity-free restriction)

$$
\operatorname{dim} \operatorname{Hom}_{H}\left(\left.\pi\right|_{H}, \tau\right) \leq 1
$$

for any irreducible smooth representation $\pi$ of $G$ and for any irreducible smooth representation $\tau$ of $H$.

Clearly, we have $(\mathrm{MF}) \Rightarrow(\mathrm{BM}) \Rightarrow(\mathrm{FM})$. Fact 1.8 is summarized by the following equivalences in the vertical direction:

$$
(\mathrm{MF}) \quad \Rightarrow \quad(\mathrm{BM}) \Rightarrow(\mathrm{FM}) \quad \cdots \text { Representation Theory }
$$

[13, Theorem D] $\mathbb{1} \mathbb{1 3}$, Theorem C]

$$
(\mathrm{BB}) \Rightarrow(\mathrm{PP}) \quad \cdots \text { Geometry of flag varieties }
$$

We note that the properties (FM) and (BM) depend only on the Lie algebras $(\mathfrak{g}, \mathfrak{h})$. Moreover, the bounded-multiplicity property (BM) depends only on the complexified Lie algebra $\left(\mathfrak{g}_{\mathbb{C}}, \mathfrak{h}_{\mathbb{C}}\right)$, as was proved in 13 . On the other hand, the multiplicityfree property $(\mathrm{MF})$ is not determined by the pair of Lie algebras $(\mathfrak{g}, \mathfrak{h})$, but depends on the groups $G$ and $H$. For example, the best constant $C=2$ if $(G, H)=$ $(S L(2, \mathbb{R}), S O(1,1))$ and $C=1$ if $\left(G^{\prime}, H^{\prime}\right)=(O(2,1), O(1,1))$ although the Lie algebras $(\mathfrak{g}, \mathfrak{h})$ and $\left(\mathfrak{g}^{\prime}, \mathfrak{h}^{\prime}\right)$ are isomorphic to each other.

As a corollary of Fact 1.8 and Proposition 4.3, we have a classification of symmetric pairs $(\mathfrak{g}, \mathfrak{h})$ satisfying the property $(\mathrm{BM})$ :

Corollary 9.4. Suppose $(\mathfrak{g}, \mathfrak{h})$ is a real reductive symmetric pair. Then the following three conditions are equivalent: 
(i) For any real reductive Lie groups $G \supset H$ with Lie algebras $\mathfrak{g} \supset \mathfrak{h}$, respectively, the pair $(G, H)$ satisfies the bounded multiplicity property (BM) for restriction.

(ii) There exists a pair of (possibly disconnected) real reductive Lie groups $G \supset H$ such that $(G, H)$ satisfies the multiplicity-free property $(\mathrm{MF})$ for restriction.

(iii) The pair of the Lie algebras $(\mathfrak{g}, \mathfrak{h})$ is isomorphic (up to outer automorphisms) to the direct sum of pairs (A), (B) and (F1) - (F5).

The implication (ii) $\Rightarrow$ (i) is obvious as mentioned. The equivalence (i) $\Leftrightarrow$ (iii) is given in [13, Theorem D]. The implication (iii) $\Rightarrow$ (ii) was proved in Sun-Zhu [20]. (Thus there are two different proofs for the implication (iii) $\Rightarrow$ (i).) As a more refined form of the implication (iii) $\Rightarrow$ (ii), Gross and Prasad [6] formulated a conjecture about the restriction of an irreducible admissible tempered representation of an inner form $G$ of the group $O(n)$ over a local field to a subgroup which is an inner form $O(n-1)$ (cf. (F2) and (F4) for the Archimedean field).

Example 9.5. For the pair $(G, H)=(O(n+1,1), O(n, 1))$, the space $\operatorname{Hom}_{H}\left(\left.\pi\right|_{H}, \tau\right)$ of continuous $H$-homomorphisms was classified in [16] for all spherical principal series representations $\pi$ and $\tau$ of $G$ and $H$, respectively. This corresponds to a special case of (F5) in Corollary 9.4. The classification was based on the explicit orbit decomposition [16, Chapter 5]

$$
G \backslash(G \times G) /\left(P_{G} \times P_{G}\right) \simeq P_{G} \backslash G / P_{G},
$$

and a meromorphic family of $H$-intertwining operators were constructed for each orbit.

\subsection{Invariant trilinear forms}

A special case of a symmetric pair is the group case

$$
(G, H)=\left(G^{\prime} \times G^{\prime}, \operatorname{diag} G^{\prime}\right),
$$

for which the branching problem deals with the decomposition of the tensor product of two irreducible representations of the group $G^{\prime}$.

Furthermore, the pair $\left(G^{\prime} \times G^{\prime}, \operatorname{diag} G^{\prime}\right)$ satisfies (PP) if and only if the homogeneous space $\left(G^{\prime} \times G^{\prime} \times G^{\prime}\right) / \operatorname{diag} G^{\prime}$ is a real spherical variety in view of the following isomorphism:

$$
\left(P_{G^{\prime}} \times P_{G^{\prime}} \times P_{G^{\prime}}\right) \backslash\left(G^{\prime} \times G^{\prime} \times G^{\prime}\right) / \operatorname{diag} G^{\prime} \simeq\left(P_{G^{\prime}} \times P_{G^{\prime}}\right) \backslash\left(G^{\prime} \times G^{\prime}\right) / P_{G^{\prime}} .
$$

By these observations, we can interpret Theorem 9.1 and Corollary 9.4 in the following form (cf. [10]): 
Corollary 9.6. Suppose $G$ is a simple Lie group. Then the following three conditions on $G$ are equivalent:

(i) For any triple of admissible smooth representations $\pi_{1}, \pi_{2}$, and $\pi_{3}$ of $G$,

$$
\operatorname{dim} \operatorname{Hom}_{G}\left(\pi_{1} \otimes \pi_{2}, \pi_{3}\right)<\infty .
$$

(ii) For any triple of admissible smooth representations $\pi_{1}, \pi_{2}$ and $\pi_{3}$ of $G$, the space of invariant trilinear forms is finite-dimensional:

$$
\operatorname{dim} \operatorname{Hom}_{G}\left(\pi_{1} \otimes \pi_{2} \otimes \pi_{3}, \mathbb{C}\right)<\infty
$$

(iii) Either $G$ is compact or $\mathfrak{g}$ is isomorphic to $\mathfrak{o}(n, 1)(n \geq 2)$.

Corollary 9.7. Suppose $G$ is a simple Lie group. Then the following three conditions on $G$ are equivalent:

(i) There exists a constant $C<\infty$ such that

$$
\operatorname{dim} \operatorname{Hom}_{G}\left(\pi_{1} \otimes \pi_{2}, \pi_{3}\right) \leq C,
$$

for any irreducible smooth representations $\pi_{1}, \pi_{2}$, and $\pi_{3}$ of $G$.

(ii) There exists a constant $C<\infty$ such that

$$
\operatorname{dim} \operatorname{Hom}_{G}\left(\pi_{1} \otimes \pi_{2} \otimes \pi_{3}, \mathbb{C}\right) \leq C,
$$

for any irreducible smooth representations $\pi_{1}, \pi_{2}$, and $\pi_{3}$ of $G$.

(iii) The Lie algebra $\mathfrak{g}$ is isomorphic to one of $\mathfrak{s u}(2) \simeq \mathfrak{o}(3), \mathfrak{s u}(1,1) \simeq \mathfrak{s l}(2, \mathbb{R}) \simeq$ $\mathfrak{o}(2,1)$ or $\mathfrak{s l}(2, \mathbb{C}) \simeq \mathfrak{o}(3,1)$.

Built on the nice properties in Corollary 9.6, a meromorphic family of invariant trilinear forms of principal series representations of the Lorentz group $O(n, 1)$ was studied in 四.

\section{References}

[1] M. Berger, Les espaces symétriques non compacts, Ann. Sci. École Norm. Sup. 74 (1957), 85-177. 
[2] Y. Benoist, Multiplicité un pour les espaces symétriques exponentiels, Mém. Soc. Math. France (N.S.) No. 15 (1984), 1-37.

[3] A. Cooper, The classifying ring of groups whose classifying ring is commutative, MIT Thesis (1975), unpublished.

[4] J.-L. Clerc, T. Kobayashi, B. Ørsted, and M. Pevzner, Generalized BernsteinReznikov integrals, Math. Ann. 349 (2011), 395-431.

[5] E. B. Dynkin, Semisimple subalgebras of semisimple Lie algebras, Amer. Math. Soc. Transl. 6 (1957), 111-244.

[6] B. Gross, D. Prasad, On the decomposition of a representations of $\mathrm{SO}_{n}$ when restricted to $\mathrm{SO}_{n-1}$, Canad. J. Math. 44 (1992), 974-1002.

[7] Harish-Chandra, Representations of semisimple Lie groups on a Banach space, Proc. Nat. Acad. Sci. U. S. A. 37 (1951), 170-173.

[8] B. Kimelfeld, Homogeneous domains on flag manifolds, J. Math. Anal. \& Appl. 121, (1987), pp. 506-588.

[9] T. Kobayashi, Discrete decomposability of the restriction of $A_{\mathfrak{q}}(\lambda)$ with respect to reductive subgroups and its applications, Invent. Math. 117 (1994), 181-205; Part II, Ann. of Math. (2) 147 (1998), 709-729; Part III, Invent. Math. 131 (1998), 229-256.

[10] - Introduction to harmonic analysis on real spherical homogeneous spaces, Proceedings of the 3rd Summer School on Number Theory "Homogeneous Spaces and Automorphic Forms" in Nagano (F. Sato, ed.), 1995, 22-41 (in Japanese).

[11] _ F-method for symmetry breaking operators, Differential Geom. Appl. 33 (2014), pp. 272-289, Special issue in honour of M. Eastwood, DOI:10.1016/j.difgeo.2013.10.003. Published online 20 November 2013, (available at arXiv:1303.3545).

[12] _ Shintani functions, real spherical manifolds, and symmetry breaking operators, preprint, 36 pages, arXiv:1401.0117.

[13] T. Kobayashi, T. Oshima, Finite multiplicity theorems for induction and restriction, Adv. Math. 248 (2013), 921-944, (available at arXiv:1108.3477). 
[14] T. Kobayashi, Y. Oshima, Classification of discretely decomposable $A_{\mathfrak{q}}(\lambda)$ with respect to reductive symmetric pairs, Adv. Math., 231 (2012), 2013-2047.

[15] _ Classification of symmetric pairs with discretely decomposable restrictions of $(\mathfrak{g}, K)$-modules, Crelles Journal, published on line 13 July, 2013. 19 pp. doi:10.1515/crelle-2013-0045.

[16] T. Kobayashi, B. Speh, Intertwining operators and the restriction of representations of rank one orthogonal groups, C. R. Acad. Sci. Paris, Ser. I, 352 (2014), 89-94; Symmetry breaking for representations of rank one orthogonal groups, 131 pages, arXiv:1310.3213.

[17] M. Krämer, Multiplicity free subgroups of compact connected Lie groups, Arch. Math. (Basel) 27 (1976), 28-36.

[18] T. Oshima, J. Sekiguchi, Eigenspaces of invariant differential operators on an affine symmetric space, Invent. Math. 57 (1980), 1-81.

[19] _ The restricted root system of a semisimple symmetric pair, Adv. Stud. Pure Math., 4 (1984), 433-497.

[20] B. Sun, C.-B. Zhu, Multiplicity one theorems: the Archimedean case, Ann. of Math. 175 (2012), 23-44.

[21] N. R. Wallach, Real reductive groups. I, II, Pure and Applied Mathematics, 132 Academic Press, Inc., Boston, MA, 1988. xx+412 pp. 